\title{
Local-global principles for tori over arithmetic curves
}

\author{
Jean-Louis Colliot-Thélène, David Harbater, Julia Hartmann, \\ Daniel Krashen, Raman Parimala and Venapally Suresh
}

\begin{abstract}
In this paper, we study local-global principles for tori over semi-global fields, which are one-variable function fields over complete discretely valued fields. In particular, we show that for principal homogeneous spaces for tori over the underlying discrete valuation ring, the obstruction to a local-global principle with respect to discrete valuations can be computed using methods coming from patching. We give a sufficient condition for the vanishing of the obstruction, as well as examples where the obstruction is nontrivial or even infinite. A major tool is the notion of a flasque resolution of a torus.
\end{abstract}

\section{Introduction}

Classical local-global principles assert the existence of rational points on varieties over a global field $F$ under the assumption that the variety has points over certain overfields of $F$, which are typically obtained via completions. In recent years, such principles have also been studied over semi-global fields. A semi-global field is a one-variable function field $F$ over a complete discretely valued field $K$, that is, a finitely generated extension of $K$ of transcendence degree 1 in which $K$ is algebraically closed. See, for example, [CTHH ${ }^{+} 19$, CTPS12, CTPS16, HS16, HHK09, HHK11, HHK15a, HHK15b, Hu17].

In [HHK09], using patching techniques, a local-global principle was proven for torsors under a linear algebraic group $G$ over a semi-global field $F$, under the hypothesis that $G$ is connected and rational as an $F$-variety. The question of whether such a local-global principle also holds without any rationality hypothesis remained open until the paper [CTPS16], where counterexamples were given for $G=T$ a suitable nonrational torus. In that example, $F$ does not have a smooth projective model over the valuation ring $R$ of the complete discretely valued field $K$, and the torus $T$ over $F$ does not extend to a torus over any regular projective model of $F$ over $R$. This raised the following question, given a regular projective $R$-curve $\mathscr{X}$ with function field $F$ : If one restricts attention to $F$-groups $G$ that are the restriction of reductive $\mathscr{X}$-group schemes or of

Received 26 June 2019, accepted in final form 28 November 2019.

2010 Mathematics Subject Classification 11E72, 12G05, 14G05 (primary), 14H25, 20G15, $14 \mathrm{G} 27$ (secondary).

Keywords: linear algebraic groups, torsors, tori, local-global principles, Galois cohomology, semi-global fields, patching, flasque resolutions.

This journal is (C) Foundation Compositio Mathematica 2020. This article is distributed with Open Access under the terms of the Creative Commons Attribution Non-Commercial License, which permits non-commercial reuse, distribution, and reproduction in any medium, provided that the original work is properly cited. For commercial re-use, please contact the Foundation Compositio Mathematica.

The authors were supported on an NSF collaborative FRG grant: DMS-1463733 (DH and JH), DMS-1463901 (DK), DMS-1463882 (RP and VS). Additional support was provided by NSF collaborative FRG grant DMS1265290 (DH), NSF RTG grant DMS-1344994 and NSF grant DMS-1902144 (DK), NSF DMS-1760413 (DH and JH), NSF DMS-1401319 (RP), NSF DMS-1301785 (VS), and NSF DMS-1801951 (RP and VS). 


\section{JLCT, DH, JH, DK, RP AND VS}

reductive $R$-group schemes, do we have a local-global principle? If not, can we understand the precise obstruction to the local-global principle?

In the present text, we give answers to these questions in the case of tori. Unlike global fields, semi-global fields admit several natural collections of overfields due to the richer geometry, and hence there are several different possible local-global principles to consider. The corresponding obstructions are measured by Tate-Shafarevich groups. In our situation, for an $\mathscr{X}$-torus $T$, we show that the obstruction groups to these different local-global principles all coincide with the Tate-Shafarevich group $\amalg(F, T)$ defined with respect to the completions of $F$ at discrete valuations (see Theorem 4.4). As a result, in order to prove a local-global principle with respect to these completions of $F$, it suffices to prove such a principle with respect to finitely many overfields arising from patching. Combining this with the notion of a flasque resolution of $T$, we are able to give a double coset formula in terms of the Galois cohomology of a flasque torus $S$ (see Theorem 3.1).

TheOrem. Let $F$ be a semi-global field over a complete discretely valued field with valuation ring $R$, and let $T$ be a torus over $R$. Let $1 \rightarrow S \rightarrow Q \rightarrow T \rightarrow 1$ be a flasque resolution of $T$. Then there is an isomorphism of abelian groups

$$
\amalg(F, T) \simeq \prod_{U} H^{1}\left(F_{U}, S\right) \backslash \prod_{(U, P)} H^{1}\left(F_{U, P}, S\right) / \prod_{P} H^{1}\left(F_{P}, S\right) .
$$

Here the fields $F_{P}, F_{U}, F_{U, P}$ form a finite inverse system of fields coming from patching (see Section 1.1).

This formula implies the finiteness of $\amalg(F, T)$ for certain residue fields $k$ for which $H^{1}(k, S)$ is always finite (Theorem 4.5), including when $k$ is finitely generated over $\mathbb{Q}$. Moreover, when combined with properties of flasque tori, it often leads to an exact computation of $\amalg(F, T)$ (see Section 6, especially Theorems 6.4 and 6.5). Another ingredient for these computations is a general graph-theoretic setup that we introduce in Section 5.

We also provide a sufficient condition for the vanishing of this obstruction in terms of the closed fiber of a normal crossings model $\mathscr{X}$ of $F$. As in [HHK15a], this condition concerns the reduction graph associated with a normal crossings model, but it is more subtle (see Section 7.1, Theorem 7.3).

Theorem. Let $K$ be a complete discretely valued field with valuation ring $R$, and let $F$ be a semi-global field over $K$. Let $\mathscr{X}$ be a normal crossings model of $F$, and assume that the associated reduction graph $\Gamma$ is a monotonic tree. Then for any $R$-torus $T$, the group $\amalg(F, T)$ is trivial.

In Section 8.2, we give examples where the obstruction is nontrivial, and in one case even infinite. Finally, in Section 8.3, we give an example in which the obstruction vanishes even though one might have expected it to be nontrivial.

\section{Background on patching}

In this section, we recall the main ingredients from patching and their use in the study of local-global principles over semi-global fields. We begin by recalling the patching setup [HHK09, Notation 3.3]. 


\section{LOCAL-GLOBAL PRINCIPLES FOR TORI}

\subsection{The patching setup}

Let $R$ be a complete discrete valuation ring, $K$ its field of fractions and $k$ its residue field. Let $t$ denote a uniformizing parameter for $R$. Let $F$ be a semi-global field over $K$. A normal model of $F$ is an integral $R$-scheme $\mathscr{X}$ with function field $F$ that is flat and projective over $R$ of relative dimension 1 and that is normal as a scheme. We write $X$ for the closed fiber $\mathscr{X} \times_{R} k$. If $\mathscr{X}$ is a normal model which is regular as a scheme, we say that $\mathscr{X}$ is a regular model. Such a regular model exists by the main theorem in [Lip78]. In fact, by [Lip75, §1, p. 193], there exists a regular model $\mathscr{X}$ for which the reduced closed fiber $X^{\text {red }}$ is a union of regular curves, with normal crossings. We call such a model a normal crossings model of $F$.

Let $\mathscr{P}$ be a finite nonempty set of closed points of $X$ that contains all the points of $X^{\text {red }}$ at which different components meet. Let $\mathscr{U}$ be the set of irreducible components of $X^{\text {red }} \backslash \mathscr{P}$. Note that $X^{\text {red }} \backslash \mathscr{P}$ is an affine curve. For such a component $U \in \mathscr{U}$, we define $F_{U}$ to be the field of fractions of the $t$-adic completion $\widehat{R}_{U}$ of the ring $R_{U} \subset F$ consisting of the rational functions on $\mathscr{X}$ that are regular at all points of $U$. Note that $\widehat{R}_{U}$ is $I$-adically complete for the radical $I$ of the ideal generated by $t$ in $\widehat{R}_{U}$. The quotient $\widehat{R}_{U} / I$ equals $k[U]$, the ring of regular functions on the integral affine curve $U$. For a (not necessarily closed) point $P$ of $X$, we let $F_{P}$ denote the field of fractions of the complete local ring $\widehat{R}_{P}:=\widehat{\mathcal{O}}_{\mathscr{X}, P}$ of $\mathscr{X}$ at $P$.

Let $U \in \mathscr{U}$, and let $P \in \mathscr{P}$ be a closed point that is in the closure $\bar{U}$ of $U$ inside $X$ (recall that $\bar{U}$ is a regular curve). The pair $(U, P)$ is a branch of $X$ at $P$ on $U$. Let $\widehat{R}_{U, P}$ denote the completion of the localization of $\widehat{\mathcal{O}}_{\mathscr{X}, P}$ at the codimension 1 point that is associated with the generic point of $\bar{U}$. This is a discrete valuation ring; let $F_{U, P}$ denote its fraction field. There is an inclusion $F_{P} \subset F_{U, P}$ induced by the inclusion $\widehat{R}_{P} \subset \widehat{R}_{U, P}$. There is also an inclusion $F_{U} \subset F_{U, P}$, induced by the inclusion $\widehat{R}_{U} \rightarrow \widehat{R}_{U, P}$. (See [HHK11, beginning of Section 4].)

In the later sections, we will also need to consider residue fields and constant fields. For a point $P \in X$, we let $\kappa(P)$ denote its residue field. For an affine open $U \subseteq X$, we define $\kappa(U)=\mathcal{O}(\bar{U})$, the ring of functions on the closure of $U$; this is the field of constants of the irreducible component $\bar{U}$ of $X^{\text {red }}$. Note that there is a natural inclusion of fields $\kappa(U) \rightarrow \kappa(P)$ when $P$ is on the closure of $U$.

\subsection{Obstructions to local-global principles over semi-global fields}

Let $G$ be a linear algebraic group over a semi-global field $F$, that is, a smooth affine group scheme of finite type over $F$. In this subsection, we define various collections of overfields of $F$, the associated local-global principles for $G$-torsors, and their obstructions, which are subsets of the Galois cohomology set $H^{1}(F, G)$.

Recall that a $G$-torsor $Z$ over $F$ is called trivial if it is isomorphic to $G$ as a $G$-space (with the action given by translation); equivalently, $Z$ has an $F$-point. Isomorphism classes of $G$ torsors over $F$ correspond bijectively to the elements of the pointed set $H^{1}(F, G)$, and under this identification, the trivial torsor corresponds to the trivial element in $H^{1}(F, G)$.

Given a collection of overfields $\left(F_{i}\right)_{i \in I}$ of $F$, one can consider the corresponding local-global principle for rational points on $G$-torsors: Must a $G$-torsor $Z$ which has a point over each $F_{i}$ also have an $F$-point? Equivalently, must an element in the kernel of $H^{1}(F, G) \rightarrow \prod_{i \in I} H^{1}\left(F_{i}, G\right)$ be the trivial class? Consequently, the kernel of the local-global map describes the obstruction to a local-global principle for rational points for all $G$-torsors over $F$.

The first such obstruction set we consider is defined via discrete valuations, in analogy to the number field case. Let $\Omega$ be the set of discrete valuations of $F$. For $v \in \Omega$, let $F_{v}$ be the 
completion of $F$ at $v$.

We define

$$
\amalg(F, G)=\operatorname{ker}\left(H^{1}(F, G) \rightarrow \prod_{v \in \Omega} H^{1}\left(F_{v}, G\right)\right) ;
$$

here the kernel is defined as the preimage of the trivial element.

The other obstruction sets we consider are defined using a normal crossings model $\mathscr{X}$ of the semi-global field $F$. As above, let $X$ denote the closed fiber of $\mathscr{X}$. We then define

$$
\amalg_{X}(F, G)=\operatorname{ker}\left(H^{1}(F, G) \rightarrow \prod_{P \in X} H^{1}\left(F_{P}, G\right)\right),
$$

where $P$ runs through all the points of $X$ (including generic points of components of $X$ ).

For $\mathscr{P}$ a subset of the reduced closed fiber and corresponding $\mathscr{U}$ as above, we let

$$
\amalg_{\mathscr{P}}(F, G)=\operatorname{ker}\left(H^{1}(F, G) \rightarrow \prod_{\zeta \in \mathscr{U} \cup \mathscr{P}} H^{1}\left(F_{\zeta}, G\right)\right) .
$$

Finally, let $\mathscr{X}^{(1)}$ be the set of codimension 1 points of $\mathscr{X}$, and let

$$
\amalg_{\mathscr{X}}(F, G)=\operatorname{ker}\left(H^{1}(F, G) \rightarrow \prod_{x \in \mathscr{X}^{(1)}} H^{1}\left(F_{x}, G\right)\right) .
$$

Here for $x \in \mathscr{X}^{(1)}$, we write $F_{x}$ for the fraction field of the complete local ring at $x$ (which is the same as the completion of $F$ at the discrete valuation of $F$ given by $x$ ).

There are several known containments among these obstruction sets. Namely,

$$
\amalg_{\mathscr{P}}(F, G) \subseteq \amalg_{X}(F, G) \subseteq \amalg(F, G) \subseteq \amalg_{\mathscr{X}}(F, G) .
$$

The first inclusion was shown in [HHK15a, Corollary 5.9], the second one is in [HHK15a, Proposition 8.2], and the final one is by definition. One also has $\cup_{\mathscr{P}} \amalg_{\mathscr{P}}(F, G)=\amalg_{X}(F, G)[\mathrm{HHK} 15 \mathrm{a}$, Corollary 5.9]; the union is taken over all subsets $\mathscr{P}$ that satisfy the conditions above.

If $G$ is a reductive group over the scheme $\mathscr{X}$ (rather than merely over $F$ ), then by [CTPS12, Theorem 4.2(ii)], one further has

$$
\cup_{\mathscr{P}} \amalg_{\mathscr{P}}(F, G)=\amalg_{X}(F, G)=\amalg(F, G)=\amalg_{\mathscr{X}}(F, G) .
$$

(In loc. cit., it was assumed that the group is defined over the underlying discrete valuation ring $R$, but the proof of part (ii) in fact only relies on $G$ being defined over $\mathscr{X}$.) For tori, a strengthening of this is given at Theorem 4.4 below.

The definitions here are given for a general linear algebraic group $G$; in this manuscript, we study these obstruction sets when $G$ is a torus. In that case, the Galois cohomology sets are abelian groups, and the obstruction sets are subgroups.

Although completions with respect to discrete valuations are in closest analogy to classical local-global principles, the geometrically defined obstruction set $\amalg_{\mathscr{P}}(F, G)$ is easier to compute explicitly (and as noted above, they are equal in interesting cases). In particular, we recall the following theorem.

ThEOREM 1.1 ([HHK15a, Corollary 3.6]). For any linear algebraic group $G$ over $F$, we have a bijection of pointed sets

$$
\amalg_{\mathscr{P}}(F, G) \simeq \prod_{U} G\left(F_{U}\right) \backslash \prod_{(U, P)} G\left(F_{U, P}\right) / \prod_{P} G\left(F_{P}\right) .
$$




\section{LOCAL-GLOBAL PRINCIPLES FOR TORI}

Here the left and right products run over $\mathscr{U}$ and $\mathscr{P}$, respectively, and the middle product runs over the branches $(U, P)$. For a commutative group $G$, this is an isomorphism of groups.

\section{Background on R-equivalence and flasque tori}

In this section, we recall results from [CTS77] and [CTS87] (to which we refer, including for the history of these results).

Let $k$ be a field, and let $k_{s}$ be a separable algebraic closure of $k$. A $k$-torus is an algebraic group $T$ over $k$ such that $T \times_{k} k_{s}$ is isomorphic to a product of copies of $\mathbb{G}_{m, k_{s}}$. Its character group is the group of homomorphisms of $k_{s}$-algebraic groups $\hat{T}=\operatorname{Hom}_{k_{s} \text {-gp }}\left(T \times_{k} k_{s}, \mathbb{G}_{m, k_{s}}\right)$. It is a $\operatorname{Gal}\left(k_{s} / k\right)$-lattice, that is, a free finitely generated abelian group with a continuous discrete action of $\operatorname{Gal}\left(k_{s} / k\right)$. This $\operatorname{Gal}\left(k_{s} / k\right)$-lattice determines the $k$-torus. The $k$-torus is said to be split by a Galois extension $\ell / k$ if $T \times_{k} \ell$ is $\ell$-isomorphic to a product of copies of $\mathbb{G}_{m, \ell}$.

A quasitrivial $k$-torus $Q$ is a $k$-torus which is $k$-isomorphic to a finite product $\prod_{i} R_{k_{i} / k} \mathbb{G}_{m}$, for finite separable field extensions $k_{i}$ of $k$. Such a $k$-torus $Q$ is an open subset of $\prod_{i} R_{k_{i} / k} \mathbb{G}_{a}$, which is $k$-isomorphic to an affine space $\mathbb{A}_{k}^{n}$. In particular, $Q$ is a rational linear algebraic group. Shapiro's lemma and Hilbert's Theorem 90 imply that the Galois cohomology group $H^{1}(k, Q)$ of a quasitrivial $k$-torus $Q$ is trivial. These properties are stable under field extensions of $k$.

A flasque $k$-torus $S$ is a $k$-torus such that $H^{1}\left(H, \operatorname{Hom}_{\mathbb{Z}}(\hat{S}, \mathbb{Z})\right)=0$ for all closed subgroups $H$ of $\operatorname{Gal}\left(k_{s} / k\right)$.

Given any $k$-torus $T$, there exists an exact sequence of $k$-tori

$$
1 \rightarrow S \rightarrow Q \rightarrow T \rightarrow 1
$$

with $Q$ quasitrivial and $S$ flasque (see [CTS77, CTS87], where the idea is attributed to Voskresenskiu). The short exact sequence is called a flasque resolution of $T$. (The torus $S$ in such a sequence is well defined up to taking a product with a quasitrivial torus.)

By taking Galois cohomology or étale cohomology, we obtain from such a flasque resolution an exact sequence

$$
Q(k) \rightarrow T(k) \rightarrow H^{1}(k, S) \rightarrow 0
$$

since $H^{1}(k, Q)=0$, as recalled above.

Another way of interpreting $H^{1}(k, S)$ is in terms of R-equivalence. Let $Z$ be a $k$-variety. Two $k$-points $P, Q \in Z(k)$ are called elementary linked if there exist an open subset $U$ of the affine line $\mathbb{A}_{k}^{1}$ and a $k$-morphism $f: U \rightarrow Z$ such that $P$ and $Q$ are in $f(U(k))$. One then defines R-equivalence on $Z(k)$ as the equivalence relation generated by this relation. If $Z=G$ is an algebraic group over $k$, the set of $k$-points that are R-equivalent to $1 \in G(k)$ is a normal subgroup. The quotient is denoted by $G(k) / \mathrm{R}$.

Now if $Q$ is a quasitrivial torus, then since $Q$ is an open subset of affine space, $Q(k) / \mathrm{R}=1$. For a flasque resolution $1 \rightarrow S \rightarrow Q \rightarrow T \rightarrow 1$, we thus have an isomorphism $T(k) / \operatorname{Im}(Q(k)) \simeq$ $H^{1}(k, S)$ and a surjection $T(k) / \operatorname{Im}(Q(k)) \rightarrow T(k) / \mathrm{R}$. In fact (see [CTS87, Theorem 3.1]), the latter map is an isomorphism, and we have

$$
T(k) / \mathrm{R} \simeq H^{1}(k, S) .
$$

For any noetherian scheme $Z$, one defines tori over $Z$, quasitrivial tori over $Z$, flasque tori over $Z$, and flasque resolutions of tori over $Z$ in an analogous way; the latter always exist. These 
notions are functorially contravariant with respect to any morphism $Y \rightarrow Z$ (see, for example, [CTS87, Proposition 1.4] for the pullback of flasque resolutions).

In this paper, a torus $T$ over $X$ is by definition isotrivial. Namely, there exists a finite étale surjective map $Y \rightarrow X$ such that $T \times_{X} Y$ is a split torus over $Y$; that is, it is isomorphic to a power $\mathbb{G}_{m, Y}^{r}$ of the multiplicative group $\mathbb{G}_{m, Y}$. We refer to [CTS87, $\S 0$ ] for basic definitions and properties of tori over a scheme.

We will use the following basic properties.

Properties 2.1. (a) Let $A$ be a regular local ring with fraction field $L$ and residue field $k$, and let $T$ be an $A$-torus. Then the natural restriction map of étale cohomology groups $H_{\text {ét }}^{1}(A, T) \rightarrow$ $H^{1}(L, T)$ is injective (by [CTS87, Theorem 4.1(i)]). If $A$ is complete, then the restriction map $H_{\text {ét }}^{1}(A, T) \rightarrow H^{1}(k, T)$ is an isomorphism ([Dem65, Proposition 8.1], [Mil80, Chapter III, Remark $3.11])$.

(b) ([CTS87, Theorem 2.2(i)]) If $S$ is a flasque torus over a regular connected scheme $Z$, then for any open set $U \subset Z$, the restriction map $H_{\text {ét }}^{1}(Z, S) \rightarrow H_{\text {ét }}^{1}(U, S)$ is surjective. Moreover, the restriction map $H_{\text {ét }}^{1}(Z, S) \rightarrow H^{1}(L, S)$ is also surjective, where $L$ is the function field of $Z$. (This condition motivates the terminology "flasque", in analogy with the term "flasque sheaf".)

(c) ([CTS87, Corollary 2.6]) If $k$ is a field, $S$ is a flasque $k$-torus, and if $U$ is a nonempty open subset of $\mathbb{A}_{k}^{n}$, the composition $H^{1}(k, S) \rightarrow H_{\text {ét }}^{1}\left(\mathbb{A}_{k}^{n}, S\right) \rightarrow H_{\text {ét }}^{1}(U, S)$ is an isomorphism.

Thus if $A$ is a regular local ring with fraction field $L$ and residue field $k$ and $S$ is a flasque torus over $A$, then there is a specialization map $H^{1}(L, S) \rightarrow H^{1}(k, S)$ that is given by

$$
H^{1}(L, S) \stackrel{\simeq}{\longleftarrow} H_{\text {ét }}^{1}(A, S) \rightarrow H^{1}(k, S),
$$

which is an isomorphism if $A$ is complete.

In particular, there is a specialization map in the case of a discrete valuation ring $A$, such as the local ring at a closed point on a regular curve with function field $L$. In the case that $A$ is a complete regular local ring of dimension 2 with residue field $k$ and $\pi$ is a regular prime of $A$ (that is, $A /(\pi)$ is regular), after Lemma 4.1 below we also give a specialization map $H^{1}\left(L_{\pi}, S\right) \rightarrow H^{1}(k, S)$ which is compatible with the specialization map $H^{1}(L, S) \rightarrow H^{1}(k, S)$ (here $L_{\pi}$ denotes the completion of $L$ with respect to $\pi$ ).

\section{The case of $F$-tori}

In this section, we give a new double coset description of $\amalg_{\mathscr{P}}(F, T)$ when $T$ is an $F$-torus. This description can be stated using a flasque resolution or using R-equivalence.

TheOREm 3.1. Let $F$ be a semi-global field, let $\mathscr{X}$ be a normal crossings model of $F$ with closed fiber $X$, and let $\mathscr{P} \subset X^{\text {red }}$ and $\mathscr{U}$ be as in Section 1.1. Let $T$ be an F-torus, and let $1 \rightarrow S \rightarrow Q \rightarrow T \rightarrow 1$ be a flasque resolution of $T$ over $F$.

(a) There is an isomorphism of abelian groups

$$
\amalg_{\mathscr{P}}(F, T) \simeq \prod_{U} H^{1}\left(F_{U}, S\right) \backslash \prod_{(U, P)} H^{1}\left(F_{U, P}, S\right) / \prod_{P} H^{1}\left(F_{P}, S\right) .
$$


(b) There is an isomorphism of abelian groups

$$
\amalg_{\mathscr{P}}(F, T) \simeq \prod_{U} T\left(F_{U}\right) / \mathrm{R} \backslash \prod_{(U, P)} T\left(F_{U, P}\right) / \mathrm{R} / \prod_{P} T\left(F_{P}\right) / \mathrm{R},
$$

where $\mathrm{R}$ denotes $\mathrm{R}$-equivalence.

Here in each double coset decomposition, the left and right products run over $\mathscr{U}$ and $\mathscr{P}$, respectively, and the middle product runs over the branches $(U, P)$.

Proof. Since $Q$ is quasitrivial, $H^{1}(F, Q)=0$. In particular, $\amalg_{\mathscr{P}}(F, Q)=0$. By Theorem 1.1 applied to $Q$, this implies that the natural map $\prod_{U} Q\left(F_{U}\right) \times \prod_{P} Q\left(F_{P}\right) \rightarrow \prod_{(U, P)} Q\left(F_{U, P}\right)$ is onto. Applying cohomology and using the vanishing of $H^{1}(L, Q)$ for any overfield $L$ of $F$, we obtain the commutative diagram of exact sequences

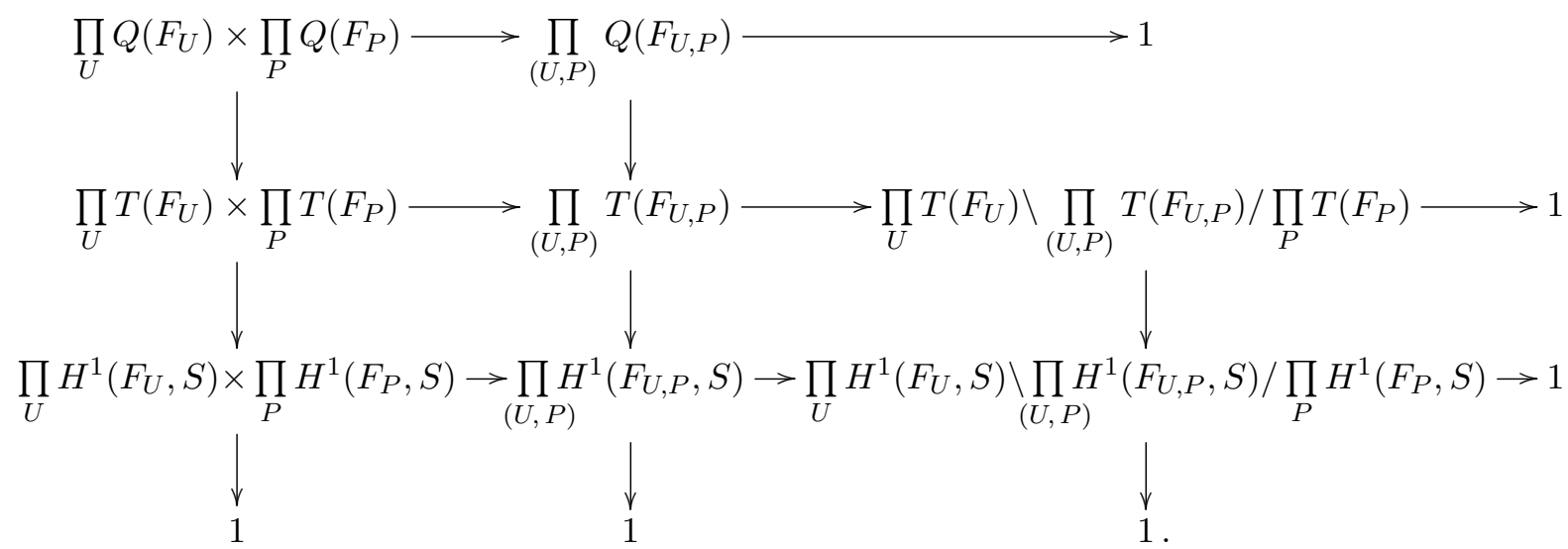

A diagram chase gives that the map

$$
\prod_{U} T\left(F_{U}\right) \backslash \prod_{(U, P)} T\left(F_{U, P}\right) / \prod_{P} T\left(F_{P}\right) \rightarrow \prod_{U} H^{1}\left(F_{U}, S\right) \backslash \prod_{(U, P)} H^{1}\left(F_{U, P}, S\right) / \prod_{P} H^{1}\left(F_{P}, S\right)
$$

is an isomorphism. By Theorem 1.1 applied to $T$, there is an isomorphism

$$
\amalg_{\mathscr{P}}(F, T) \simeq \prod_{U} T\left(F_{U}\right) \backslash \prod_{(U, P)} T\left(F_{U, P}\right) / \prod_{P} T\left(F_{P}\right) .
$$

This proves part (a). Part (b) then follows from part (a) and the functorial isomorphisms $T(L) / \mathrm{R} \simeq H^{1}(L, S)$ for any field extension $L$ of $F$.

As a first application, we obtain a finiteness result.

THEOREM 3.2. Let $k$ be either a field that is finitely generated over $\mathbb{Q}$ or a local field of characteristic zero, and let $K=k((t))$. Let $F$ be a semi-global field over $K$ with normal crossings model $\mathscr{X}$ and closed fiber $X$. Let $\mathscr{P} \subset X^{\text {red }}$ and $\mathscr{U}$ be as in Section 1.1. Then for any F-torus T, the group $\amalg_{\mathscr{P}}(F, T)$ is finite.

Proof. We claim that for each $(U, P)$ and any flasque torus $S$, the group $H^{1}\left(F_{U, P}, S\right)$ is finite. Since there are only finitely many pairs $(U, P)$, Theorem 3.1 will then imply the finiteness of $\amalg_{\mathscr{P}}(F, T)$. 


\section{JLCT, DH, JH, DK, RP AND VS}

Each field $F_{U, P}$ is of the shape $\ell((u))((t))$, with $\ell$ a finite field extension of $k$. By [CTGP04, Theorem 3.2], if $L$ is a field of characteristic zero such that $H^{1}(L, S)$ is finite for every flasque torus $S$ over $L$, then $H^{1}(L((t)), S)$ is finite for every flasque torus $S$ over $L((t))$. So to prove our claim, it suffices to show that $H^{1}(\ell, S)$ is finite for every flasque torus $S$. By assumption, $\ell$ is either finitely generated over $\mathbb{Q}$ or local. Finiteness in the former case follows from [CTS77, Theorem 1, p. 192]. Finiteness in the latter case is well known for any connected linear algebraic group (see, for example, [PR94, Theorem 6.14]).

See [CTGP04, Theorem 3.4] and the remark following it for further examples of fields $L$ with the property that $H^{1}(L, S)$ is finite for every flasque torus $S$.

\section{The case of $\mathscr{X}$-tori}

In this section, the torus under consideration is defined over a normal crossings model $\mathscr{X}$ of the semi-global field. We first show that the different obstruction sets all coincide in that case. In the second part, we give a description of the terms occurring in the double coset formula Theorem 3.1.

\subsection{Comparing various Tate-Shafarevich groups}

In this subsection, we prove a local factorization result for tori defined over a normal crossings model of the semi-global field. Using this, we show that the different obstruction sets defined in Section 1.2 all coincide.

LemMa 4.1. Let $A$ be a complete regular local ring of dimension 2 with residue field $k$ and field of fractions $L$. Let $L_{\pi}$ be the completion of $L$ at a regular prime $\pi$ of $A$. Moreover, let $T$ be a torus over $A$, and let $1 \rightarrow S \rightarrow Q \rightarrow T \rightarrow 1$ be a flasque resolution over $A$. Let $\theta: H^{1}\left(L_{\pi}, S\right) \rightarrow H^{1}(k, S)$ be the composition of the specialization maps $H^{1}\left(L_{\pi}, S\right) \rightarrow H^{1}(k(\pi), S) \rightarrow H^{1}(k, S)$. Then $\theta$ is an isomorphism, and the diagram

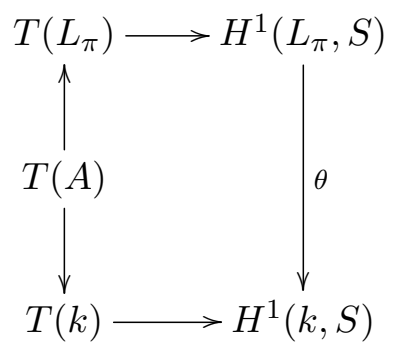

commutes. Here the horizontal maps are the coboundary maps in the cohomology sequence coming from the flasque resolution.

Proof. The two specialization maps $H^{1}\left(L_{\pi}, S\right) \rightarrow H^{1}(k(\pi), S)$ and $H^{1}(k(\pi), S) \rightarrow H^{1}(k, S)$ are isomorphisms by the completeness of the discrete valuation rings $\widehat{A}_{(\pi)}$ and $A /(\pi)$, where the former ring is the completion of the localization of $A$ at $\pi$; see the comment after Properties 2.1. Thus $\theta$ is an isomorphism. For the commutativity of the diagram, recall that the above two specialization maps are respectively given by

$$
\begin{aligned}
H^{1}\left(L_{\pi}, S\right) & \simeq H_{\text {ét }}^{1}\left(\widehat{A}_{(\pi)}, S\right) \rightarrow H^{1}(k(\pi), S), \\
H^{1}(k(\pi), S) & \simeq H_{\text {ét }}^{1}(A /(\pi), S) \rightarrow H^{1}(k, S) .
\end{aligned}
$$




\section{LOCAL-GLOBAL PRINCIPLES FOR TORI}

The commutativity now easily follows using that the compositions $T(A) \rightarrow T\left(\widehat{A}_{(\pi)}\right) \rightarrow T(k(\pi))$ and $T(A) \rightarrow T(A /(\pi)) \rightarrow T(k(\pi))$ define the same maps and that the coboundary map $T(\cdot) \rightarrow$ $H^{1}(\cdot, S)$ is functorial.

The isomorphism $\theta$, which is a composition of two specialization maps, is compatible with the specialization map $H^{1}(L, S) \rightarrow H^{1}(k, S)$ described after Properties 2.1. That is, the latter map is the same as the composition $H^{1}(L, S) \rightarrow H^{1}\left(L_{\pi}, S\right) \stackrel{\theta}{\rightarrow} H^{1}(k, S)$ since the restriction maps to the residue fields are isomorphisms; see Properties 2.1(a). We thus also call $\theta$ the specialization map from $H^{1}\left(L_{\pi}, S\right)$ to $H^{1}(k, S)$.

Now let $F$ be a semi-global field with normal crossings model $\mathscr{X}$ and closed fiber $X$. Let $\mathscr{P}$ and $\mathscr{U}$ be as in Section 1.1. We next use the specialization map to prove a factorization lemma.

Lemma 4.2. Let $T$ be a torus over $\mathscr{X}$. Let $1 \rightarrow S \rightarrow Q \stackrel{\varphi}{\rightarrow} T \rightarrow 1$ be a flasque resolution of $T$ over $\mathscr{X}$. Let $U$ be a nonempty affine open subset of an irreducible component of the reduced closed fiber $X^{\text {red }}$ of $\mathscr{X}$ such that $U$ does not meet any other components of $X^{\text {red }}$. Then for any $P$ in the complement $\bar{U} \backslash U$ of $U$ in its closure, $T\left(F_{U, P}\right)=T\left(\widehat{R}_{P}\right) \varphi\left(Q\left(F_{U, P}\right)\right)$.

Proof. Consider the diagram

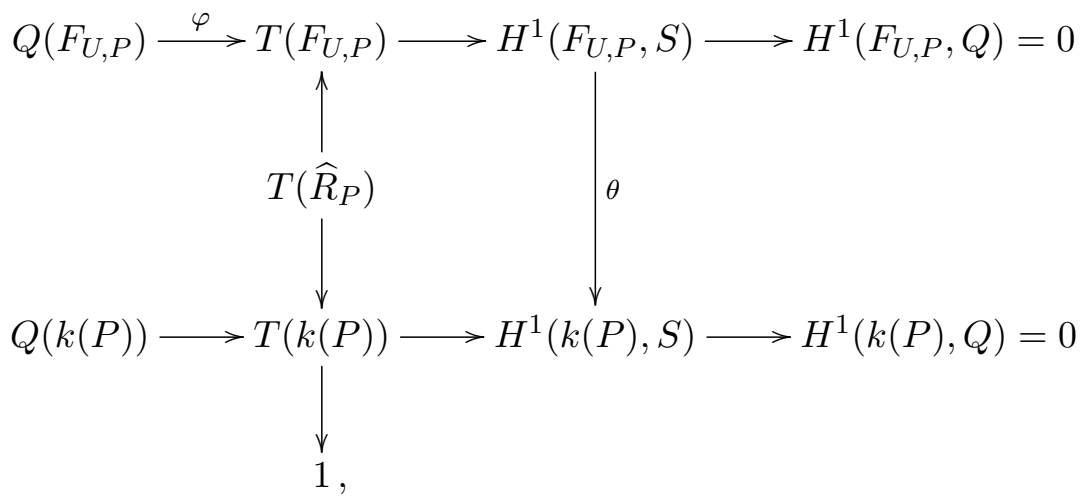

where $\theta$ is the isomorphism given by Lemma 4.1. The two cohomology groups on the right vanish since $Q$ is quasitrivial. The assertion now follows from an easy diagram chase.

Proposition 4.3. Let $F$ be a semi-global field with normal crossings model $\mathscr{X}$, and let $T$ be a torus over $\mathscr{X}$. Let $U$ be a nonempty affine open subset of an irreducible component of the reduced closed fiber $X^{\text {red }}$ of $\mathscr{X}$ such that $U$ does not meet any other components of $X^{\text {red }}$. Let $P \in U$ be a closed point and $V=U \backslash\{P\}$. Then $\operatorname{ker}\left(H^{1}\left(F_{U}, T\right) \rightarrow H^{1}\left(F_{V}, T\right) \times H^{1}\left(F_{P}, T\right)\right)$ is trivial.

Proof. Let $1 \rightarrow S \rightarrow Q \rightarrow T \rightarrow 1$ be a flasque resolution of $T$ over the ring $\widehat{R}_{P}$ defined in Section 1.1 (we continue to use $T$ to denote the restriction of $T$ over $\widehat{R}_{P}$ ). By Lemma 4.2 applied to $P$ and $V$, any element of $T\left(F_{V, P}\right)$ is the product of an element of $T\left(\widehat{R}_{P}\right) \subset T\left(F_{P}\right)$ and an element in the image of $Q\left(F_{V, P}\right)$. Since $Q$ is a quasitrivial $F$-torus, $Q$ is rational, and thus $Q\left(F_{V, P}\right)=Q\left(F_{V}\right) Q\left(F_{P}\right)$ by [HHK15b, Proposition 3.9 and Corollary 3.15]. Thus $T\left(F_{V, P}\right)=$ $T\left(F_{V}\right) T\left(F_{P}\right)$. By [HHK15b, Theorem 2.13 and Proposition 3.9], this concludes the proof.

The next theorem shows that in the case of tori, it is sufficient to consider $\amalg_{\mathscr{P}}(F, T)$ for some $\mathscr{P}$ (rather than taking the union over all possible $\mathscr{P}$ ). 


\section{JLCT, DH, JH, DK, RP AND VS}

Theorem 4.4. Let $F$ be a semi-global field with normal crossings model $\mathscr{X}$, and let $\mathscr{P}$ be as in Section 1.1. Let $T$ be a torus over $\mathscr{X}$. Then the subgroups $\amalg_{\mathscr{P}}(F, T), \amalg_{X}(F, T), \amalg(F, T)$, and $\amalg_{\mathscr{X}}(F, T)$ of $H^{1}(F, T)$ all coincide.

Proof. By the chain of equalities given just before Theorem 1.1, it suffices to show that $\amalg_{X}(F, T)$ $\subseteq \amalg_{\mathscr{P}}(F, T)$. So let $\zeta \in \amalg_{X}(F, T)$. Then $\zeta \otimes F_{P}$ is trivial for every point $P \in X$ by definition (including generic points of components of $X$ ). If $U \in \mathscr{U}$, then by [HHK15a, Proposition 5.8], there exists a nonempty open subset $V$ of $U$ such that $\zeta \otimes F_{V}$ is trivial. Since $U \backslash V$ is a finite set, by Proposition 4.3, the class $\zeta \otimes F_{U}$ is trivial. Hence $\zeta \in \amalg_{\mathscr{P}}(F, T)$.

Combining this with Theorem 3.2, we immediately obtain the following.

THEOREM 4.5. Let $k$ be either a finitely generated field over $\mathbb{Q}$ or a local field of characteristic zero, and let $K=k((t))$. Let $F$ be a semi-global field over $K$ with normal crossings model $\mathscr{X}$, and let $\mathscr{P}$ be as in Section 1.1. Let $T$ be a torus over $\mathscr{X}$. Then the groups $\amalg_{\mathscr{P}}(F, T), \amalg_{X}(F, T)$, $\amalg(F, T)$, and $\amalg_{\mathscr{X}}(F, T)$ are all finite.

Proof. By Theorem 3.2, the group $\amalg_{\mathscr{P}}(F, T)$ is finite. The result then follows from Theorem 4.4.

See Example 8.7 for an example where $\amalg(F, T)$ is infinite.

\subsection{On the value of $\amalg_{\mathscr{P}}(F, T)$ when $T$ is an $\mathscr{X}$-torus}

For a torus $T$ over a semi-global field $F$, Theorem 3.1 gives a formula for $\amalg_{\mathscr{P}}(F, T)$ in terms of a flasque resolution of $T$.

If $T$ is an $\mathscr{X}$-torus and $1 \rightarrow S \rightarrow Q \rightarrow T \rightarrow 1$ is a flasque resolution of $T$ over $\mathscr{X}$, pullback induces a flasque resolution of $T$ over $F$. In that situation, we now analyze the maps $H^{1}\left(F_{P}, S\right) \rightarrow H^{1}\left(F_{U, P}, S\right)$ and $H^{1}\left(F_{U}, S\right) \rightarrow H^{1}\left(F_{U, P}, S\right)$ which were relied on in the formula in Theorem 3.1(a).

In what follows, we abuse notation: For a $Z$-torus $S$ and a morphism of schemes $Y \rightarrow Z$, we let $H^{1}(Y, S)$ denote the étale cohomology group $H_{\text {ét }}^{1}\left(Y, S_{Y}\right)$, where $S_{Y}$ is the $Y$-torus $S \times_{Z} Y$. For an affine scheme $Z=\operatorname{Spec} A$, we write $H^{1}(Z, S)=H^{1}(A, S)$.

Recall that we denote the residue field at a point $P \in X$ by $\kappa(P)$.

Proposition 4.6. Let $F$ be a semi-global field with normal crossings model $\mathscr{X}$, and let $\mathscr{P}$ and $\mathscr{U}$ be as in Section 1.1. Let $S$ be a flasque torus over $\mathscr{X}$.

(a) For each point $P \in \mathscr{P}$, the specialization map $H^{1}\left(F_{P}, S\right) \rightarrow H^{1}(\kappa(P), S)$ is an isomorphism.

(b) For each branch $(U, P)$, the specialization map $\theta: H^{1}\left(F_{U, P}, S\right) \rightarrow H^{1}(\kappa(P), S)$ from Lemma 4.1 is an isomorphism that is compatible with the isomorphism in part (a), in the sense that we have a commutative diagram

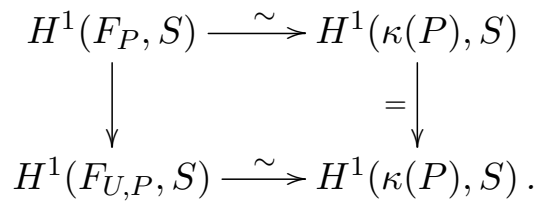

(c) For each branch $(U, P)$, consider the map

$$
H^{1}\left(F_{U}, S\right) \rightarrow H^{1}\left(F_{U, P}, S\right) \stackrel{\sim}{\rightarrow} H^{1}(\kappa(P), S)
$$


induced by the inclusion $F_{U} \subset F_{U, P}$. For a fixed $U \in \mathscr{U}$, the images of the following maps coincide:

- $H^{1}\left(F_{U}, S\right) \rightarrow \prod_{(U, P)} H^{1}\left(F_{U, P}, S\right) \stackrel{\sim}{\rightarrow} \prod_{(U, P)} H^{1}(\kappa(P), S)$

- the product of the restriction maps $H^{1}(\bar{U}, S) \rightarrow \prod_{(U, P)} H^{1}(\kappa(P), S)$

- the product of the specialization maps $H^{1}(k(U), S) \rightarrow \prod_{(U, P)} H^{1}(\kappa(P), S)$.

Here $\bar{U}$ denotes the closure of $U$, and the products are taken over all branches $(U, P)$ at points $P \in \mathscr{P}$ on $\bar{U}$.

Proof. Part (a) follows from the completeness of the local ring $\widehat{R}_{P}$, by Properties 2.1. For part (b), it was shown in Lemma 4.1 that $\theta$ is an isomorphism; the commutativity of the diagram follows from the compatibility stated just after the proof of that lemma (taking $L=F_{P}$ and $L_{\pi}=F_{U, P}$ ). For part (c), consider the commutative diagram

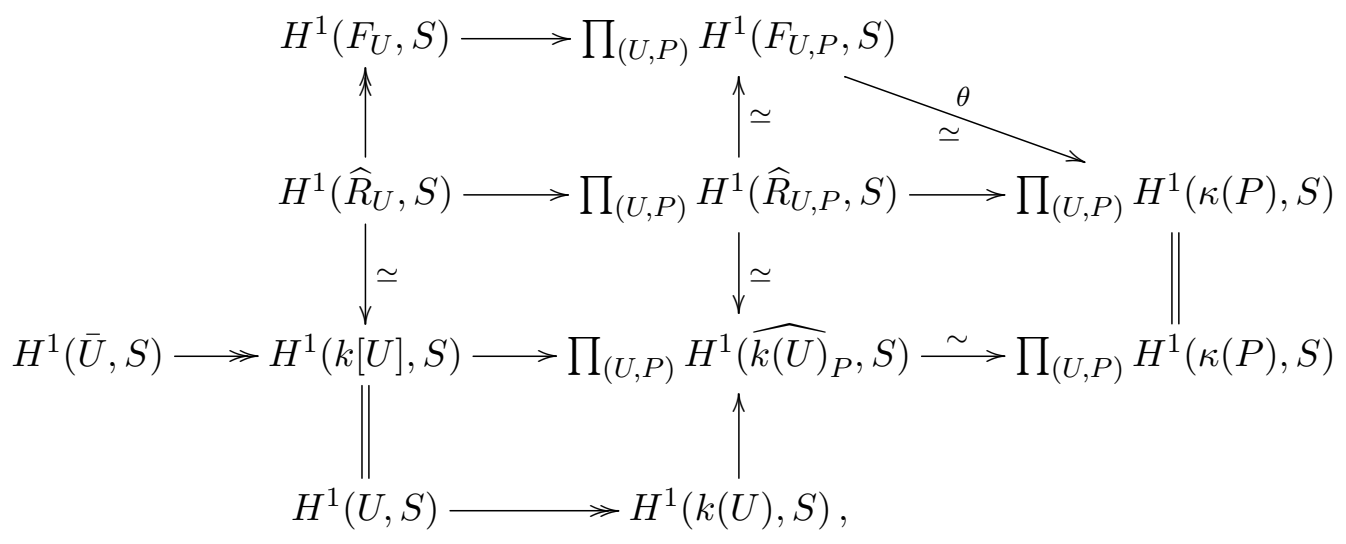

where $\widehat{k(U)}_{P}$ is the completion of $k(U)=k(\bar{U})$ at the discrete valuation associated with $P$. Here the two vertical maps from $\prod_{(U, P)} H^{1}\left(\widehat{R}_{U, P}, S\right)$ are isomorphisms by Properties 2.1. For each $P$, the composition $H^{1}\left(F_{U, P}, S\right) \rightarrow H^{1}\left(\widehat{k(U)}_{P}, S\right)$ and the map $H^{1}\left(\widehat{k(U)}_{P}, S\right) \rightarrow H^{1}(\kappa(P), S)$ are both specialization isomorphisms. The map $H^{1}\left(\widehat{R}_{U}, S\right) \rightarrow H^{1}(k[U], S)$ is also an isomorphism because $\widehat{R}_{U}$ is complete with respect to an ideal $I$ such that $\widehat{R}_{U} / I=k[U]$; see [Str83, Theorem 1]. Three other maps in the above diagram are surjections, as indicated, by Properties 2.1(b). It then follows from this diagram that the three maps considered in part (c) each have the same image as the map $H^{1}(k[U], S) \rightarrow \prod_{(U, P)} H^{1}(\kappa(P), S)$.

The following corollary requires the flasque torus $S$ to be defined over the underlying discrete valuation ring $R$ rather than just over $\mathscr{X}$. A group scheme $S$ over the complete discrete valuation ring $R$ induces $S_{\mathscr{X}}$ over $\mathscr{X}$ by pullback, as well as its restrictions to subschemes of $\mathscr{X}$. We will continue to drop the subscripts if these group schemes appear as coefficients in cohomology groups.

Recall that for an affine open $U \subseteq X$, we define $\kappa(U)=\mathcal{O}(\bar{U})$, the ring of functions on the closure of $U$, and that $\kappa(U)$ naturally embeds into $\kappa(P)$ when $P$ is a point on the closure of $U$.

Corollary 4.7. Let $K$ be a complete discretely valued field with ring of integers $R$, let $F$ be a semi-global field over $K$ with normal crossings model $\mathscr{X}$, and let $\mathscr{P}$ and $\mathscr{U}$ be as in Section 1.1. Let $S$ be a flasque $R$-torus.

(a) Let $(U, P)$ be a branch. If $\kappa(U)=\kappa(P)$, then the map $H^{1}(\bar{U}, S) \rightarrow H^{1}(\kappa(P), S)$ is surjective, hence so is the map $H^{1}\left(F_{U}, S\right) \rightarrow H^{1}(\kappa(P), S)$. 
(b) Assume that each component of the closed fiber $X$ of $\mathscr{X}$ is $k$-isomorphic to $\mathbb{P}_{k}^{1}$. Let $U \in \mathscr{U}$, and let $P_{1}, \ldots, P_{n}$ be the closed points of $\mathscr{P}$ which lie on $\bar{U}$. Then the image of the map $H^{1}\left(F_{U}, S\right) \rightarrow \prod_{j=1}^{n} H^{1}\left(\kappa\left(P_{j}\right), S\right)$ coincides with the image of the diagonal map

$$
H^{1}(k, S) \rightarrow \prod_{j=1}^{n} H^{1}\left(\kappa\left(P_{j}\right), S\right) .
$$

Proof. (a) We have an inclusion morphism $i: P \rightarrow \bar{U}$, and by the hypothesis, we also have a structure morphism $j: \bar{U} \rightarrow \operatorname{Spec}(\kappa(P))=P$. The composition $j i$ is the identity. Taking cohomology, we obtain $i^{*}: H^{1}(\bar{U}, S) \rightarrow H^{1}(P, S)$ and $j^{*}: H^{1}(P, S) \rightarrow H^{1}\left(\bar{U}, j^{*}(S)\right)=H^{1}(\bar{U}, S)$. The last equality follows from the fact that $S$ is defined over $\bar{U}$ via pullback from $R$, together with the fact that the map $\bar{U} \rightarrow \operatorname{Spec}(k) \subset \operatorname{Spec}(R)$ factors through $P$ by hypothesis. Since $j i$ is the identity, the composition

$$
H^{1}(\kappa(P), S) \rightarrow H^{1}(\bar{U}, S) \rightarrow H^{1}(\kappa(P), S)
$$

is also the identity map, yielding the first assertion. The second assertion follows from the first one by the last assertion of Proposition 4.6(c).

(b) By Proposition 4.6(c), the image of $H^{1}\left(F_{U}, S\right) \rightarrow \prod_{j=1}^{n} H^{1}\left(\kappa\left(P_{j}\right), S\right)$ coincides with the image of $H^{1}(\bar{U}, S) \rightarrow \prod_{j=1}^{n} H^{1}\left(\kappa\left(P_{j}\right), S\right)$. If $k$ is finite, the statement is clear since all the $H^{1}\left(\kappa\left(P_{j}\right), S\right)$ vanish (use the Lang isogeny, [Lan56, Theorem 2]). If $k$ is not finite, let $V \subset \bar{U}$ be an open subset isomorphic to $\mathbb{A}_{k}^{1}$ containing all points $P_{j}$. Then the map $H^{1}(\bar{U}, S) \rightarrow$ $\prod_{j=1}^{n} H^{1}\left(\kappa\left(P_{j}\right), S\right)$ factors through $H^{1}(V, S)=H^{1}\left(\mathbb{A}_{k}^{1}, S\right)=H^{1}(k, S)$ by Properties $2.1(\mathrm{c})$, and the assertion follows.

\section{Decorated graphs and contraction results}

For a semi-global field $F$ and an $F$-torus $T$, we will obtain results about $\amalg(F, T)$ by considering a graph associated with the closed fiber of a normal crossings model of $F$. In this section, we will establish the graph-theoretic prerequisites for this study.

\subsection{Decorated graphs}

When we use the term graph, we will allow multiple edges between vertices as well as loops. It will be convenient notationally to consider every edge as having two distinct "ends" or "halves", each of which will be attached to a vertex (possibly the same one). All graphs will be assumed finite.

Given a graph $\Gamma$ with vertex set $V$ and edge set $E$, and an edge $e$ connecting distinct vertices $x$ and $y$, we can form a new graph $\Gamma / e$, called the contraction of $\Gamma$ along $e$, by removing the edge $e$ and identifying the vertices $x$ and $y$ (denoting the resulting vertex by $[x y]$ ). More formally, $\Gamma / e$ is the graph with vertex set $V^{\prime}=(V \backslash\{x, y\}) \cup\{[x y]\}$ and edge set $E^{\prime}=E \backslash\{e\}$, where an edge $e^{\prime} \in E^{\prime}$ is adjacent to $v^{\prime} \in V^{\prime} \backslash\{[x y]\}$ if $e^{\prime}$ is adjacent to $v^{\prime}$ in $\Gamma$, and where $e^{\prime}$ is adjacent to [xy] in $\Gamma^{\prime}$ if $e^{\prime}$ is adjacent to either $x$ or $y$ in $\Gamma$.

Let $\Gamma$ be a graph. A coefficient system $A$ on $\Gamma$ is a rule that associates abelian groups $A_{e}$ and $A_{x}$ with each edge $e$ and vertex $x$, and associates a homomorphism $A_{\alpha}: A_{x} \rightarrow A_{e}$ with each half edge $\alpha$ of $e$ that is attached to $x$. We refer to a pair $(\Gamma, A)$ consisting of a graph $\Gamma$ and a coefficient system $A$ on $\Gamma$ as a decorated graph.

If $A$ and $B$ are coefficient systems on the same graph $\Gamma$, we define a morphism $A \rightarrow B$ to be a collection of group homomorphisms $A_{x} \rightarrow B_{x}$ and $A_{e} \rightarrow B_{e}$ which commute with the 


\section{LOCAL-GLOBAL PRINCIPLES FOR TORI}

maps $A_{\alpha}$. Further, we can form the kernel and cokernel of a morphism componentwise and obtain an abelian category - these are, in fact, just the abelian categories of morphisms from a diagram category coming from the graph $\Gamma$ to the category of abelian groups.

\subsection{Cohomology of decorated graphs}

Given a coefficient system $A$ on a graph $\Gamma$, we write $\mathcal{C}(\Gamma, A)$ for the cochain complex

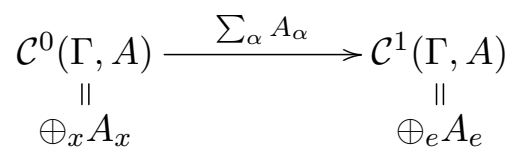

concentrated in degrees 0 and 1 . Note that this gives a faithful (though not necessarily full) exact functor from the abelian category of coefficient systems on $\Gamma$ to the abelian category of abelian complexes. We define $H^{i}(\Gamma, A)=H^{i}(\mathcal{C}(\Gamma, A))$.

Lemma 5.1. Suppose that we have a graph $\Gamma$ and a short exact sequence of coefficient systems $0 \rightarrow A \rightarrow B \rightarrow C \rightarrow 0$ on $\Gamma$. Then we obtain a 6 -term exact sequence of cohomology

$$
0 \rightarrow H^{0}(\Gamma, A) \rightarrow H^{0}(\Gamma, B) \rightarrow H^{0}(\Gamma, C) \rightarrow H^{1}(\Gamma, A) \rightarrow H^{1}(\Gamma, B) \rightarrow H^{1}(\Gamma, C) \rightarrow 0 .
$$

Proof. By the exactness of the above functor, $0 \rightarrow A \rightarrow B \rightarrow C \rightarrow 0$ induces an exact sequence of complexes $0 \rightarrow \mathcal{C}(\Gamma, A) \rightarrow \mathcal{C}(\Gamma, B) \rightarrow \mathcal{C}(\Gamma, C) \rightarrow 0$ and thus a long exact sequence of cohomology groups (where the higher cohomology groups vanish since each complex is concentrated in degrees 0 and 1).

\subsection{Contraction of coefficients}

Suppose that we are given a decorated graph $(\Gamma, A)$ and that $e$ is an edge with half edges $\alpha$ and $\beta$ attached to vertices $x$ and $y$, respectively. We say that the half edge $\alpha$ is redundant if $x \neq y$ and $A_{\alpha}: A_{x} \rightarrow A_{e}$ is an isomorphism. In this case, we may define a new coefficient system $A / \alpha$ on the graph $\Gamma / e$ as follows. As before, write $V^{\prime}$ and $E^{\prime}$ for the vertices and edges of $\Gamma / e$. We set

$$
(A / \alpha)_{v}=A_{v} \text { for } v \in V^{\prime} \backslash\{[x y]\}, \quad(A / \alpha)_{[x y]}=A_{y}, \quad(A / \alpha)_{f}=A_{f} \text { for } f \in E^{\prime} .
$$

For a half edge $\gamma$ of $\Gamma$, we define the homomorphisms $(A / \alpha)_{\gamma}$ to coincide with $A_{\gamma}$ in case $\gamma$ is not attached to $x$ or $y$. If $\gamma$ is attached to $y$ in $\Gamma$ and lies on the edge $f$, we let $(A / \alpha)_{\gamma}$ be the map $A_{\gamma}: A_{y} \rightarrow A_{f}$ pre-composed with the identification of $A_{y}$ with $(A / \alpha)_{[x y]}$. Finally, if $\gamma$ is attached to $x$ in $\Gamma$, we let $(A / \alpha)_{\gamma}$ be the composition

$$
(A / \alpha)_{[x y]}=A_{y} \stackrel{A_{\beta}}{\longrightarrow} A_{e} \stackrel{-A_{\alpha}^{-1}}{\longrightarrow} A_{x} \stackrel{A_{\gamma}}{\longrightarrow} A_{f}=(A / \alpha)_{f} .
$$

The pair $(\Gamma / e, A / \alpha)$ is called the contraction of the decorated graph $(\Gamma, A)$ along $\alpha$. We say that $(\Gamma, A)$ can be contracted to a point if it can be reduced to a single vertex with no edges by performing a series of contractions of decorated graphs.

Lemma 5.2. Let $(\Gamma, A)$ and the contraction $(\Gamma / e, A / \alpha)$ be as above. Then we have a natural morphism of complexes $\pi: \mathcal{C}(\Gamma / e, A / \alpha) \rightarrow \mathcal{C}(\Gamma, A)$ inducing an isomorphism on cohomology groups.

Proof. We define the morphism $\pi$ as follows. Let $\alpha$ and $\beta$ be the half edges at $e$, attached to vertices $x$ and $y$ of $\Gamma$, respectively. For a vertex $v \notin\{x, y\}$ of $\Gamma$ and an edge $f \neq e$ of $\Gamma$, we define $\pi$ to map the groups $(A / \alpha)_{v}$ and $(A / \alpha)_{f}$ to $A_{v}$ and $A_{f}$, respectively, via the canonical 
identifications and to take $(A / \alpha)_{[x y]}=A_{y}$ to $A_{x} \oplus A_{y} \subset \mathcal{C}^{0}(\Gamma, A)$ by $a_{y} \mapsto\left(-A_{\alpha}^{-1} A_{\beta}\left(a_{y}\right), a_{y}\right)$. It is straightforward to check that this commutes with the differential - in other words, is a morphism of complexes. One can also check that the cokernel of the map $C^{0}(\Gamma / e, A / \alpha) \rightarrow$ $C^{0}(\Gamma, A)$ is exactly $A_{x}$, where the isomorphism is induced by the map $C^{0}(\Gamma, A) \rightarrow A_{x}$ given by $\left(a_{v}\right)_{v \in V} \mapsto A_{\alpha}^{-1} A_{\beta}\left(a_{y}\right)+x$.

The cokernel of the map $C^{1}(\Gamma / e, A / \alpha) \rightarrow C^{1}(\Gamma, A)$ is just $A_{e}$ via the projection map. We then obtain a map of short exact sequences

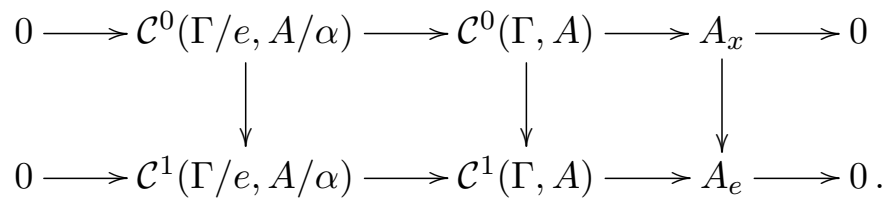

Since the vertical map on the right is an isomorphism (it is the isomorphism $A_{\alpha}$ ), the snake lemma gives an isomorphism of the kernels and cokernels of the other two vertical maps. But these are the groups $H^{0}$ and $H^{1}$ of the complexes, respectively.

The following example illustrates the effect of contracting edges and relates the cohomology of decorated graphs to that of the underlying graph as a topological space.

ExAmPLE 5.3. Let $\Gamma$ be a graph, let $A_{0}$ be an abelian group, and let $A$ be a coefficient system on $\Gamma$ in which each $A_{e}$ and each $A_{x}$ is isomorphic to $A_{0}$ and in which every $A_{\alpha}$ is an isomorphism. We may wish to compare $H^{1}(\Gamma, A)$ (which depends on the choice of the isomorphisms $A_{\alpha}$ ) with the group $H^{1}\left(\Gamma_{\text {top }}, A_{0}\right)$, where the latter group is the usual cohomology of the topological space $\Gamma_{\text {top }}$ associated with the graph $\Gamma$, viewed as a finite simplicial complex, with coefficients in the group $A_{0}$. Depending on the choice of the isomorphisms $A_{\alpha}$, these cohomology groups sometimes agree, and sometimes do not, as illustrated below.

(a) We will first observe that the topological cohomology agrees with the cohomology of decorated graphs with respect to a particular choice of isomorphisms. To see this, for each edge $e$ of $\Gamma$, choose an orientation. Now if $e$ is an edge from vertex $x$ to vertex $y$, and if $\alpha$ is the half edge on $e$ attached to $y$, define $A_{\alpha}: A_{y} \rightarrow A_{e}$ to be the identity map on the group $A_{0}$; define $A_{\beta}: A_{x} \rightarrow A_{e}$ to be $-\mathrm{id}_{A_{0}}$ for the other half edge $\beta$ (attached to $x$ ). Let $A_{\Gamma}$ be the coefficient system defined by these choices. Then $H^{1}\left(\Gamma, A_{\Gamma}\right)=H^{1}\left(\Gamma_{\text {top }}, A_{0}\right)$, by the definition of simplicial cohomology.

(b) Consider a bipartite graph, that is, a graph $\Gamma$ with vertex set partitioned as $V=V^{\prime} \sqcup V^{\prime \prime}$ such that each edge has a vertex in $V^{\prime}$ and a vertex in $V^{\prime \prime}$. In this case, the cohomology of a constant coefficient system with identity isomorphisms $A_{\alpha}$ agrees with that of the topological space. More precisely, take the coefficient system $A$, in which each edge and vertex is decorated by the group $A_{0}$ and in which each homomorphism associated with a half edge is the identity. We may also consider the orientation in which each edge is chosen to start at a vertex in $V^{\prime}$ and end in a vertex in $V^{\prime \prime}$. Let $A^{\prime}$ be the coefficient system associated with this set of choices as in part (a). Define a morphism of coefficient systems $\phi: A \rightarrow A^{\prime}$ by choosing $\phi_{x}: A_{x} \rightarrow A_{x}^{\prime}$ to be the identity if $x \in V^{\prime \prime}$ and the negative of the identity if $x \in V^{\prime}$. Since $\phi$ is an isomorphism, part (a) yields $H^{1}(\Gamma, A) \simeq H^{1}\left(\Gamma, A^{\prime}\right) \simeq$ $H^{1}\left(\Gamma_{\text {top }}, A_{0}\right)$.

(c) As an example where $H^{1}\left(\Gamma, A_{\Gamma}\right)$ and $H^{1}\left(\Gamma_{\text {top }}, A_{0}\right)$ do not agree, let $\Gamma$ be a triangle, that is, a graph consisting of three vertices $v_{1}, v_{2}, v_{3}$ and three edges $e_{1}, e_{2}, e_{3}$, where $v_{i}$ and $v_{j}$ are the endpoints of $e_{k}$ for each permutation $(i, j, k)$ of $(1,2,3)$. Let $A_{0}=\mathbb{Q}$, and let each $A_{\alpha}$ 


\section{LOCAL-GLOBAL PRINCIPLES FOR TORI}

be the identity. Then $H^{1}\left(\Gamma_{\text {top }}, A_{0}\right)=\mathbb{Q}$, but $H^{1}\left(\Gamma, A_{\Gamma}\right)=0$ by a direct computation. Note that $\Gamma$ is not bipartite, so this does not contradict part (b).

We give a sufficient condition for when a decorated graph can be contracted to a point.

Proposition 5.4. Let $(\Gamma, A)$ be a decorated graph. Assume that $\Gamma$ is a tree for which there is a choice of some vertex $v_{0}$ of $\Gamma$ (the "root") with the following property:

Suppose that $v$ and $w$ are vertices of $\Gamma$ and $v$ is the parent of $w$ via an edge $e$ (that is, $v$ and $w$ are adjacent via $e$, and $v$ lies on the unique path connecting $v_{0}$ to $\left.w\right)$. Let $\alpha$ be the half edge associated with $w$ and $e$ (there is only one since a tree has no loops). Then $A_{\alpha}: A_{w} \rightarrow A_{e}$ is an isomorphism (equivalently, the half edge $\alpha$ is redundant).

Then $(\Gamma, A)$ can be contracted to a point.

Proof. We show by induction on the number of vertices that $(\Gamma, A)$ can be contracted to a point. Let $w$ be a leaf of $(\Gamma, A)$ (that is, a vertex of degree 1), and let $v$ be its parent in $\Gamma$ via an edge $e$. Let $\alpha$ be the half edge associated with $w$. By assumption, $\alpha$ is redundant. The contraction $(\Gamma / e, A / \alpha)$ is a decorated graph with fewer vertices. These vertices are the vertices of $\Gamma$ except with $w$ removed; the decorations of those remaining vertices are unchanged. Hence $(\Gamma / e, A / \alpha)$ also satisfies the hypothesis of the proposition and so can be contracted to a point, by induction. Hence $(\Gamma, A)$ can be contracted to a point.

\section{Decorations of the reduction graph and computations of $\amalg(F, T)$}

As before, consider a complete discrete valuation $\operatorname{ring} R$ with fraction field $K$ and residue field $k$, and a semi-global field $F$ over $K$. Let $\mathscr{X}$ be a normal crossings model of $F$, and let $\mathscr{P}$ be a finite nonempty set of closed points of $\mathscr{X}$ as in Section 1.1. Associated with $X^{\text {red }}$ and $\mathscr{P}$ is the so-called reduction graph $\Gamma$, as in [HHK15a, Section 6] (see below). In this section, we apply the graph-theoretic setup from Section 5 to this graph.

\subsection{Comparison of coefficient systems}

Recall from Section 1.1 that $\mathscr{X}$ is a connected regular integral proper curve over $R$ with function field $F$ such that the reduced closed fiber $X^{\text {red }}$ is a union of connected regular $k$-curves $C_{i}$ that intersect each other transversally. Also recall that $\mathscr{P}$ is a nonempty set of closed points of $\mathscr{X}$ that contains the (possibly empty) finite set $\mathscr{P}_{0}$ of closed points at which two components $C_{i}$ and $C_{j}$ meet. Let $\mathscr{U}$ be the set of connected components of the complement of $\mathscr{P}$ in the reduced closed fiber $X^{\text {red }}$. Associated with this is the reduction graph $\Gamma=\Gamma(\mathscr{X}, \mathscr{P})$. This is a bipartite graph whose vertex set is $\mathscr{P} \cup \mathscr{U}$ and that has an edge connecting $U \in \mathscr{U}$ to $P \in \mathscr{P}$ for each branch $(U, P)$.

Remark 6.1. Recall that for a normal crossings model $\mathscr{X}$, there is also another graph that can be associated with the reduced closed fiber, namely the dual graph $\Gamma_{\mathrm{D}}$ (as defined before [DM69, Lemma 1.16, p. 86]). Its vertices correspond to the irreducible components of the closed fiber, and its edges correspond to intersection points of those irreducible components. If the closed fiber does not consist of just a single (regular) irreducible component, then the set of intersection points $\mathscr{P}_{0}$ is nonempty and the reduction graph $\Gamma$ associated with the reduced closed fiber and $\mathscr{P}_{0}$ is the barycentric subdivision of $\Gamma_{\mathrm{D}}$ (see [HHK15a, Remark 6.1(a)]). Even if $\mathscr{P}$ is strictly larger than $\mathscr{P}_{0}$, the graphs $\Gamma_{\mathrm{D}}$ and $\Gamma$ are homotopy equivalent as topological spaces. 
Associated with each $P, U$, and $(U, P)$, we have fields $F_{P}, F_{U}$, and $F_{U, P}$ (see Section 1.1). For each $P$, we also have the residue fields $\kappa(P)$ of $\mathscr{X}$ at $P$. Recall that for $U \in \mathscr{U}$, we defined $\kappa(U)=\mathcal{O}(\bar{U})$, the ring of functions on the closure of $U$. Let $S$ be a flasque torus over $R$. If $P$ is in the closure of $U$, there is a specialization map $H^{1}(k(U), S) \rightarrow H^{1}(\kappa(P), S)$ (defined after Properties 2.1). We define the following coefficient systems:

(a) $\mathcal{H}_{F}(S)$ : Each vertex $\xi$ in $\mathscr{P} \cup \mathscr{U}$ is decorated with $H^{1}\left(F_{\xi}, S\right)$, and each edge $(U, P)$ is decorated with $H^{1}\left(F_{U, P}, S\right)$. The homomorphisms for the half edges are induced by the inclusions $F_{P}, F_{U} \hookrightarrow F_{U, P}$.

(b) $\mathcal{H}_{k}(S)$ : Each vertex $P$ in $\mathscr{P}$ is decorated with $H^{1}(\kappa(P), S)$, each vertex $U$ in $\mathscr{U}$ is decorated with $H^{1}(k(U), S)$, and each edge $(U, P)$ is decorated with $H^{1}(\kappa(P), S)$. The homomorphisms for the half edges are the identity (for $P$ ) and the specialization map (for $U$ ).

(c) $\mathcal{H}_{\kappa}(S)$ : Each vertex $\xi$ in $\mathscr{P} \cup \mathscr{U}$ is decorated with $H^{1}(\kappa(\xi), S)$, and each edge $(U, P)$ is decorated with $H^{1}(\kappa(P), S)$. The homomorphisms for the half edges are induced by the identity (for $P$ ) and the natural inclusion map $\kappa(U) \rightarrow \kappa(P)$.

Lemma 6.2. Let $T$ be an $R$-torus, and let $1 \rightarrow S \rightarrow Q \rightarrow T \rightarrow 1$ be a flasque resolution of $T$. Then $\amalg(F, T) \simeq H^{1}\left(\Gamma, \mathcal{H}_{F}(S)\right) \simeq H^{1}\left(\Gamma, \mathcal{H}_{k}(S)\right)$.

Proof. By Theorem 3.1,

$$
\amalg_{\mathscr{P}}(F, T) \simeq \prod_{U} H^{1}\left(F_{U}, S\right) \backslash \prod_{(U, P)} H^{1}\left(F_{U, P}, S\right) / \prod_{P} H^{1}\left(F_{P}, S\right) .
$$

The left-hand side equals $\amalg(F, T)$ by Theorem 4.4. The right-hand side equals $H^{1}\left(\Gamma, \mathcal{H}_{F}(S)\right)$ by definition.

For the second identification, we note that by Proposition 4.6(a,b), we may identify $H^{1}\left(F_{P}, S\right)$ $\simeq H^{1}(\kappa(P), S)$ and $H^{1}\left(F_{U, P}, S\right) \simeq H^{1}(\kappa(P), S)$. Since $H^{1}\left(\Gamma, \mathcal{H}_{F}(S)\right)$ and $H^{1}\left(\Gamma, \mathcal{H}_{k}(S)\right)$ are therefore cokernels of homomorphisms to the isomorphic groups $\prod_{(U, P)} H^{1}\left(F_{U, P}, S\right)$ and $\prod_{(U, P)} H^{1}(\kappa(P), S)$, we need only show that the images of these homomorphisms coincide. But this follows from the fact that by Proposition 4.6(b), we have a commutative diagram

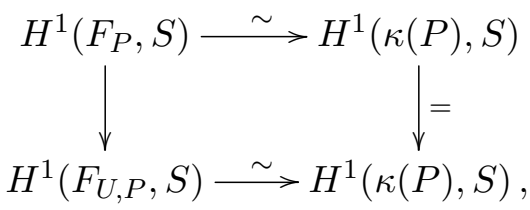

and from the fact that the images of $H^{1}\left(F_{U}, S\right)$ and $H^{1}(k(U), S)$ in $\prod_{(U, P)} H^{1}(\kappa(P), S)$ coincide by Proposition 4.6(c).

\subsection{Applications to $\amalg(\boldsymbol{F}, \boldsymbol{T})$}

Proposition 6.3. Let $T$ be an $R$-torus, and let $1 \rightarrow S \rightarrow Q \rightarrow T \rightarrow 1$ be a flasque resolution of $T$.

(a) There is a natural surjection

$$
\phi: H^{1}\left(\Gamma, \mathcal{H}_{\kappa}(S)\right) \rightarrow H^{1}\left(\Gamma, \mathcal{H}_{F}(S)\right)=\amalg(F, T) .
$$

(b) If $A$ is a constant coefficient system with value $H^{1}(\ell, S)$ on each marking, where $\ell / k$ is a finite field extension, then

$$
H^{1}(\Gamma, A)=H^{1}\left(\Gamma_{\text {top }}, H^{1}(\ell, S)\right)=\operatorname{Hom}\left(H_{1}\left(\Gamma_{\text {top }}, \mathbb{Z}\right), H^{1}(\ell, S)\right),
$$




\section{LOCAL-GLOBAL PRINCIPLES FOR TORI}

where $\Gamma_{\text {top }}$ denotes the topological space associated with $\Gamma$.

(c) If every component of the closed fiber $X$ of $\mathscr{X}$ is isomorphic to a projective line (over its field of constants), then $\phi$ is an isomorphism.

Proof. By Lemma 6.2, we have that $\amalg(F, T) \simeq H^{1}\left(\Gamma, \mathcal{H}_{F}(S)\right) \simeq H^{1}\left(\Gamma, \mathcal{H}_{k}(S)\right)$. But there is a natural map of coefficient systems $\mathcal{H}_{\kappa}(S) \rightarrow \mathcal{H}_{k}(S)$ which is the identity on the decorations for the edges $(U, P)$. Since we have a commutative diagram

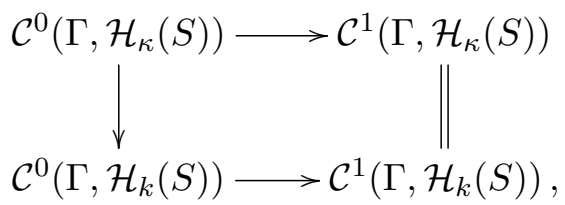

it follows that the image of the bottom map contains the image of the top map, which induces a surjective map of cokernels $H^{1}\left(\Gamma, \mathcal{H}_{\kappa}(S)\right) \rightarrow H^{1}\left(\Gamma, \mathcal{H}_{k}(S)\right)$. This proves part (a).

The second equality in part (b) is by the universal coefficient theorem, using that the coefficient system is constant. The first equality is the content of Example 5.3(b) because the reduction graph $\Gamma$ is bipartite.

Finally, for part (c), since every component is isomorphic to a projective line, we have an isomorphism $H^{1}(\kappa(U), S) \rightarrow H_{\text {ét }}^{1}(U, S)$ by Property 2.1(c) and a surjection $H_{\text {ét }}^{1}(U, S) \rightarrow$ $H^{1}(k(U), S)$ by Property 2.1(b); so the composition $H^{1}(\kappa(U), S) \rightarrow H^{1}(k(U), S)$ is surjective. In particular, if we consider the coefficient system $\mathcal{K}$ defined by the exact sequence $0 \rightarrow \mathcal{K} \rightarrow$ $\mathcal{H}_{\kappa}(S) \rightarrow \mathcal{H}_{k}(S) \rightarrow 0$, then $\mathcal{K}$ is nonzero only on markings coming from vertices, not edges. It follows that $H^{1}(\Gamma, \mathcal{K})=0$; by the long exact sequence, $H^{1}\left(\Gamma, \mathcal{H}_{\kappa}(S)\right) \simeq H^{1}\left(\Gamma, \mathcal{H}_{k}(S)\right) \simeq \amalg(F, T)$, with the second isomorphism holding by Lemma 6.2. This proves part (c).

Proposition 6.3 gives an upper bound on $\amalg(F, T)$ for $T$ an $\mathscr{X}$-torus, in terms of the constant fields of the components of the reduced closed fiber $X^{\text {red }}$ of our normal crossings model $\mathscr{X}$ and the residue fields of the intersection points of the irreducible components of $X^{\text {red }}$. In particular, we immediately obtain the following.

Theorem 6.4. Let $\mathscr{X}$ be a normal crossings model of a semi-global field $F$ over a complete discretely valued field $K$ with residue field $k$, and suppose that the reduced closed fiber $X^{\text {red }}$ consists of $k$-curves $C_{i}$ that intersect only at $k$-points. Let $m$ be the number of cycles in the reduction graph (that is, the rank of $H_{1}\left(\Gamma_{\text {top }}, \mathbb{Z}\right)$ ). Let $T$ be an $R$-torus, and let $1 \rightarrow S \rightarrow Q \rightarrow$ $T \rightarrow 1$ be a flasque resolution of $T$.

(a) The group $\amalg(F, T)$ is a quotient of $H^{1}(k, S)^{m}$.

(b) If the reduction graph is a tree, then $\amalg(F, T)$ is trivial.

(c) If each $C_{i}$ is isomorphic to $\mathbb{P}_{k}^{1}$, then $\amalg(F, T)$ is isomorphic to $H^{1}(k, S)^{m}$. Hence $\amalg(F, T)$ is trivial if and only if the reduction graph is a tree or $H^{1}(k, S)$ vanishes.

The following theorem gives a generalization of the last part of Theorem 6.4. Its proof provides a general strategy for utilizing coefficient systems.

Theorem 6.5. Let $\mathscr{X}$ be a normal crossings model of a semi-global field $F$ over a complete discretely valued field $K$ with residue field $k$, and suppose that the reduced closed fiber $X^{\text {red }}$ consists of a union of copies of $\mathbb{P}_{k}^{1}$. Let $\mathscr{P}$ be the set of intersection points (which might not be 
rational points). Let $\Gamma$ be the associated reduction graph. Then for any $R$-torus $T$ with flasque resolution $1 \rightarrow S \rightarrow Q \rightarrow T \rightarrow 1$, there is an exact sequence

$$
\operatorname{Hom}\left(H_{1}\left(\Gamma_{\text {top }}, \mathbb{Z}\right), H^{1}(k, S)\right) \rightarrow \amalg(F, T) \rightarrow \prod_{P \in \mathscr{P}} \frac{H^{1}(\kappa(P), S)}{\operatorname{im} H^{1}(k, S)} \rightarrow 0 .
$$

Proof. If $\mathscr{P}$ is empty, that is, $X^{\text {red }}=\mathbb{P}_{k}^{1}$, the claim follows from Theorem $6.4(\mathrm{c})$. So we may assume that $\mathscr{P}$ is not empty. Consider the coefficient system $\mathcal{H}_{k}(S)$. As in the proof of Proposition 6.3, one sees that there is a surjection $H^{1}(\kappa(U), S)=H^{1}(k, S) \rightarrow H^{1}(k(U), S)$ for each component $U$. By specializing to a $k$-point, one checks that this is also injective, so $H^{1}(k(U), S) \simeq H^{1}(k, S)$ for each $U$. Let $A_{\bullet}$ be the constant coefficient system given by decorating every vertex and edge with $H^{1}(k, S)$, and let $C_{\bullet}$. denote the quotient of the coefficient system $\mathcal{H}_{k}(S)$ modulo $A_{\bullet}$. Since $H^{1}(k, S)=H^{1}(k(U), S)$ for each component $U$, the system $C_{\bullet}$ has trivial decorations at such $U$. In particular, $H^{0}\left(\Gamma, C_{\bullet}\right)=0$. Let $A_{\bullet}^{\prime}$ and $A_{\bullet}^{\prime \prime}$ be the kernel and image of $A_{\bullet} \rightarrow \mathcal{H}_{k}(S)$, respectively. Applying Lemma 5.1 to $0 \rightarrow A_{\bullet}^{\prime} \rightarrow A_{\bullet} \rightarrow A_{\bullet}^{\prime \prime} \rightarrow 0$ and to $0 \rightarrow A_{\bullet}^{\prime \prime} \rightarrow \mathcal{H}_{k}(S) \rightarrow C_{\bullet} \rightarrow 0$ gives an exact sequence

$$
H^{1}\left(\Gamma, A_{\bullet}\right) \rightarrow H^{1}\left(\Gamma, \mathcal{H}_{k}(S)\right) \rightarrow H^{1}\left(\Gamma, C_{\bullet}\right) \rightarrow 0 .
$$

The middle term is $\amalg(F, T)$ by Lemma 6.2. The left term is $\operatorname{Hom}\left(H_{1}\left(\Gamma_{\text {top }}, \mathbb{Z}\right), H^{1}(k, S)\right)$ by Proposition 6.3(b). To calculate the right term, recall that $C$. has trivial decorations at components $U$. The decoration at a point $P \in \mathscr{P}$ is $H^{1}(\kappa(P), S) / H^{1}(k, S)$, and the decoration at a branch $(U, P)$ is also $H^{1}(\kappa(P), S) / H^{1}(k, S)$. The right term is the quotient of $\prod_{(P, U)}\left(H^{1}(\kappa(P), S) / H^{1}(k, S)\right)$ by $\prod_{P \in \mathscr{P}}\left(H^{1}(\kappa(P), S) / H^{1}(k, S)\right)$. But each $P$ lies on exactly two branches, so that quotient is $\prod_{P \in \mathscr{P}}\left(H^{1}(\kappa(P), S) / H^{1}(k, S)\right)$, as claimed.

This theorem will be used in Section 8 to provide explicit examples where $\amalg(F, T)$ is nontrivial.

\section{Monotonic reduction graphs and the vanishing of $\amalg(F, T)$}

We next obtain a vanishing result for $\amalg(F, T)$ for a special type of reduction graph, which we call a "monotonic tree". We also give two alternate descriptions of such trees, and we give examples to show that other natural tree-like assumptions on the reduction graph do not suffice to guarantee the vanishing of $\amalg(F, T)$.

\subsection{Vanishing of $\amalg(\boldsymbol{F}, \boldsymbol{T})$}

Let $F$ be a semi-global field with normal crossings model $\mathscr{X}$, and let $\Gamma$ be the associated reduction graph. With each vertex $v$ of the reduction graph $\Gamma$, we associate the field $\kappa(v)$. We say that the reduction graph $\Gamma$ is monotonic if it is a tree and there is a choice of some vertex $v_{0}$ (the "root") with the following property: if $v$ and $w$ are vertices of $\Gamma$ and $v$ is the parent of $w$ (that is, $v$ and $w$ are adjacent, and $v$ lies on the unique path connecting $v_{0}$ to $\left.w\right)$, then $\kappa(v) \subseteq \kappa(w)$. Recall that if $P \in \mathscr{P}$ lies on the closure of $U \in \mathscr{U}$, then $\kappa(P)$ necessarily contains $\kappa(U)=\mathcal{O}(\bar{U})$; hence if $P$ is the parent of $U$ on a monotonic tree, then necessarily $\kappa(P)=\kappa(U)$.

Remark 7.1. If $\mathscr{X}$ is a normal crossings model with associated reduction graph $\Gamma$ as above, it is easy to check that the reduction graph of any blow-up of $\mathscr{X}$ is monotonic if and only if $\Gamma$ is monotonic. For any two normal crossings models $\mathscr{X}_{i}(i=1,2)$ of $F$, there exist a normal crossings model $\mathscr{X}$ of $F$ and morphisms $\mathscr{X} \rightarrow \mathscr{X}_{i}$ which are given by a series of blow-ups. 


\section{LOCAL-GLOBAL PRINCIPLES FOR TORI}

Indeed, one first considers the closure $\mathcal{Z} \subset \mathscr{X}_{1} \times_{R} \mathscr{X}_{2}$ of the diagonal of the generic fibers. By $[\operatorname{Lip} 75, \S 1$, p. 193], there exist a normal crossings model $\mathscr{X}$ and a proper birational $R$-morphism $\mathscr{X} \rightarrow \mathcal{Z}$. By [Lic68, Theorem II.1.15], each composite morphism $\mathscr{X} \rightarrow \mathcal{Z} \rightarrow \mathscr{X}_{i}$ is a composite of blow-ups in closed points. So for a semi-global field $F$, either all associated reduction graphs are monotonic or none of them is.

Let $S$ be an $R$-torus, and let $\mathcal{H}_{\kappa}(S)$ be the coefficient system on $\Gamma$ defined in Section 6.1. From Proposition 5.4, we immediately obtain the following.

Lemma 7.2. With $F, \mathscr{X}, \Gamma$ as above, assume that $\Gamma$ is a monotonic tree. Then $\left(\Gamma, \mathcal{H}_{\kappa}(S)\right)$ can be contracted to a point.

Proof. The decorated graph $\left(\Gamma, \mathcal{H}_{\kappa}(S)\right)$ satisfies the condition in Proposition 5.4 since it is monotonic, the decoration of each edge $(U, P)$ is $H^{1}(\kappa(P), S)$ by definition, and $\kappa(P)=\kappa(U)$ if $P$ is the parent of $U$.

Theorem 7.3. Let $K$ be a complete discretely valued field with valuation ring $R$, and let $F$ be a semi-global field over $K$. Let $\mathscr{X}$ be a normal crossings model of $F$, and assume that the associated reduction graph $\Gamma$ is a monotonic tree. Then for any $R$-torus $T$, the group $\amalg(F, T)$ is trivial.

Proof. Let $1 \rightarrow S \rightarrow Q \rightarrow T \rightarrow 1$ be a flasque resolution of $T$. We then have a coefficient system $\mathcal{H}_{\kappa}(S)$ on $\Gamma$ as above. By Proposition 6.3 , there is a surjection $H^{1}\left(\Gamma, \mathcal{H}_{\kappa}(S)\right) \rightarrow \amalg(F, T)$. So it suffices to show that $H^{1}\left(\Gamma, \mathcal{H}_{\kappa}(S)\right)$ is trivial. By Lemma 7.2 , the decorated graph $\left(\Gamma, \mathcal{H}_{\kappa}(S)\right)$ can be contracted to a point. But Lemma 5.2 asserts that contraction does not change the cohomology of the graph. That is, $H^{1}\left(\Gamma, \mathcal{H}_{\kappa}(S)\right)$ is the cohomology of a (decorated) graph consisting of a single vertex and no edge, and thus it is trivial, as we wanted to show.

Remark 7.4. Note that in the situation of Theorem 6.4(b), the reduction graph is a monotonic tree, with all labels equal to $k$. Hence this illustrates Theorem 7.3.

\subsection{Characterizations of monotonic trees}

In this subsection, we give two characterizations of monotonic trees.

In the above situation, let $\mathscr{P}_{0}$ be the subset of $\mathscr{P}$ consisting of the points of $X^{\text {red }}$ where two irreducible components meet. We obtain a subgraph $\Gamma_{0}$ of $\Gamma$ having vertices $\mathscr{P}_{0} \cup \mathscr{U}$ and having an edge connecting any two vertices that are adjacent in $\Gamma$. The graph $\Gamma_{0}$ is a tree if and only if $\Gamma$ is; in that case, $\Gamma_{0}$ is monotonic if and only if $\Gamma$ is.

Proposition 7.5. Let $\mathscr{X}$ be a normal crossings model for a semi-global field $F$, and suppose that the associated reduction graph $\Gamma$ is a tree. Then $\Gamma$ is monotonic if and only if there is an injection $\psi: \mathscr{P}_{0} \rightarrow \mathscr{U}$ such that for every $P \in \mathscr{P}_{0}$, we have that $P \in \overline{\psi(P)}$ and $\kappa(P)=\kappa(\psi(P))$.

Proof. For the forward direction, note that any $P \in \mathscr{P}_{0}$ lies on exactly two irreducible components of $X$. So if $\Gamma$ is monotonic, then the vertex $P$ has exactly one parent $U \in \mathscr{U}$ and one child $U^{\prime} \in \mathscr{U}$ (that is, $P$ is the parent of $U^{\prime}$ ), with $P$ in the closure of each. As noted above, $\kappa(P)=\kappa\left(U^{\prime}\right)$. Thus we may define $\psi(P)=U^{\prime}$. This map is injective because a vertex cannot have more than one parent.

For the reverse direction, consider the subtree $\Gamma_{0}$ of $\Gamma$ defined above. This is a bipartite tree with vertex sets $\mathscr{P}_{0}$ and $\mathscr{U}$, and its terminal vertices all lie in $\mathscr{U}$. By induction, one sees that such a bipartite tree has the property that $|\mathscr{U}|=\left|\mathscr{P}_{0}\right|+1$. It follows from the injectivity of $\psi$ 
that there is a unique element $U_{0} \in \mathscr{U}$ that is not in the image of $\psi$; we will show that $\Gamma$ is monotonic with respect to the root $U_{0}$.

Take any path in $\Gamma$ beginning at $U_{0}$, with consecutive vertices $v_{0}, v_{1}, \ldots, v_{n}$, where $v_{0}=U_{0}$. Thus $v_{i} \in \mathscr{U}$ for $i$ even, and $v_{i} \in \mathscr{P}$ for $i$ odd. For $i$ odd, the two elements of $\mathscr{U}$ whose closures contain $v_{i} \in \mathscr{P}$ are $v_{i-1}$ and $v_{i+1}$. But $v_{0}=U_{0}$ is not in the image of $\psi$. So $\psi\left(v_{1}\right)=v_{2}$. Since $\psi$ is injective, it follows by induction that $\psi\left(v_{i}\right)=v_{i+1}$ for all odd $i$. Thus $\kappa\left(v_{i}\right)=\kappa\left(v_{i+1}\right)$ for $i$ odd. Also, $\kappa\left(v_{i}\right) \subseteq \kappa\left(v_{i+1}\right)$ for $i$ even since in that case $v_{i+1} \in \mathscr{P}$ is in the closure of $v_{i} \in \mathscr{U}$. Thus the monotonicity condition holds for every pair of adjacent vertices in this path. Since every pair of adjacent vertices in $\Gamma$ lies in some path that begins at $U_{0}$, the result follows.

The following proposition relates the notion of a monotonic tree to the tree property of the reduction graph for the curve and its base change to finite field extensions of the residue field. By Zariski's connectedness theorem, $X$ is geometrically connected; that is, $X_{k^{\prime}}$ is connected for any finite field extension $k^{\prime} / k$ (see [Gro61, Corollaire III.4.3.12]). Given a finite nonempty subset $\mathscr{P}$ of $X^{\text {red }}$, let $\mathscr{P}_{k^{\prime}}$ denote the subset of $X_{k^{\prime}}$ consisting of the points that lie over points of $\mathscr{P}$. The graph $\Gamma^{\prime}$ associated with $X_{k^{\prime}}$ and $\mathscr{P}_{k^{\prime}}$ is defined in the natural way, as for $X$ and $\mathscr{P}$. If $\Gamma^{\prime}$ is a tree, then so is the original reduction graph $\Gamma$ because the inverse image of any loop in $\Gamma$ would contain a loop in $\Gamma^{\prime}$. The converse is more subtle, as discussed in the following proposition (see also Example 7.7 below).

Proposition 7.6. Let $R$ be a complete discrete valuation ring with fraction field $K$ and residue field $k$, and let $\mathscr{X}$ be a normal crossings model for a semi-global field $F$ over $K$. Let $X$ be the closed fiber of $\mathscr{X}$. Let $\Gamma$ be the reduction graph associated with $X^{\text {red }}$ and a finite set $\mathscr{P} \subset X^{\text {red }}$ as above.

(a) If $\Gamma$ is a monotonic tree, then for every finite field extension $k^{\prime} / k$, the graph associated with $X_{k^{\prime}}$ and $\mathscr{P}_{k^{\prime}}$ is a tree.

(b) In part (a), suppose that each of the fields $\kappa(P)$ is a separable extension of $k$ (this holds, for example, if $k$ is of characteristic zero or, more generally, perfect). Then the converse also holds: if the graph associated with $X_{k^{\prime}}$ and $\mathscr{P}_{k^{\prime}}$ is a tree for every finite field extension $k^{\prime} / k$, then $\Gamma$ is a monotonic tree.

Proof. For part (a), let $k^{\prime} / k$ be a finite field extension, and consider the graph $\Gamma^{\prime}$ associated with the base change $X_{k^{\prime}}$ of $X$ and $\mathscr{P}_{k^{\prime}}$ as introduced above. Let $\pi: \Gamma^{\prime} \rightarrow \Gamma$ be the natural map. Since $X_{k^{\prime}}$ is connected, so is the graph $\Gamma^{\prime}$.

As before, let $\mathscr{U}$ be the set of connected components of the complement of $\mathscr{P}$ in $X^{\text {red }}$. If $U \in \mathscr{U}$, then $\bar{U}_{k^{\prime}}=\pi^{-1}(\bar{U})$ consists of a disjoint union of copies of an irreducible $k^{\prime}$-curve. Thus a point of $X_{k^{\prime}}$ can lie on at most one irreducible component of $\bar{U}_{k^{\prime}}$. Moreover, if $P \in \mathscr{P}$ lies in the closure of $U \in \mathscr{U}$, with $\kappa(P)=\kappa(U)$, and if $U^{\prime}$ is a component of $\bar{U}_{k^{\prime}}$, then there is exactly one point of $\mathscr{P}_{k^{\prime}}$ over $P$ that lies on $U^{\prime}$. Together, these observations show that if $v$ and $w$ are adjacent vertices of $\Gamma$, with $v$ the parent of $w$, and if $w^{\prime}$ is a vertex of $\Gamma^{\prime}$ lying over $w$, then there is a unique vertex $v^{\prime}$ of $\Gamma^{\prime}$ that is adjacent to $w^{\prime}$ and that lies over $v$.

Pick a vertex $v_{0}^{\prime}$ of $\Gamma^{\prime}$ over the root $v_{0}$ of $\Gamma$; that is, $\pi\left(v_{0}^{\prime}\right)=v_{0}$. Now consider any path $v_{0}^{\prime}, v_{1}^{\prime}, \ldots, v_{n}^{\prime}$ in $\Gamma^{\prime}$ starting at $v_{0}^{\prime}$. (As before, paths are assumed to have no repeated edges.) Let $v_{i}=\pi\left(v_{i}^{\prime}\right)$. Then $v_{i}$ is the parent of $v_{i+1}$ for all $i<n$ since otherwise $v_{i+1}$ would be the parent of $v_{i}$, which inductively would give $v_{i+1}=v_{i-1}$, contradicting the uniqueness assertion in the previous paragraph. Thus for every path in $\Gamma^{\prime}$ from $v_{0}^{\prime}$ to a vertex $v^{\prime}$, its image in $\Gamma$ is a path such that each vertex is the parent of its successor (this is the unique path connecting $v_{0}$ to $\pi\left(v^{\prime}\right)$ ). 


\section{LOCAL-GLOBAL PRINCIPLES FOR TORI}

By the uniqueness assertion of the previous paragraph, for each vertex $v^{\prime}$ of $\Gamma^{\prime}$, there is a unique path from $v_{0}^{\prime}$ to $v^{\prime}$ in $\Gamma^{\prime}$, and it lies over the unique path from $v_{0}$ to $\pi\left(v^{\prime}\right)$ in $\Gamma$.

Now consider any edge $e^{\prime}$ in $\Gamma^{\prime}$, with vertices $v^{\prime}, w^{\prime}$. Let $e, v, w$ be their images in $\Gamma$. We may assume that $v$ is the parent of $w$. So the unique path from $v_{0}$ to $w$ ends with the edge $e$ from $v$ to $w$; concatenating $e^{\prime}$ with the unique path from $v_{0}^{\prime}$ to $v^{\prime}$ yields the unique path from $v_{0}^{\prime}$ to $w$. Hence if $e^{\prime}$ is deleted from $\Gamma^{\prime}$, then there is no path connecting $v_{0}$ to $w^{\prime}$. Thus if any edge is deleted from $\Gamma^{\prime}$, the resulting graph is disconnected. This implies that the connected graph $\Gamma^{\prime}$ is a tree.

For part (b), let $k^{\prime}$ be a finite Galois extension of $k$ that contains all the fields $\kappa(P)$ for $P \in \mathscr{P}$. Thus $k^{\prime}$ also contains the fields $\kappa(U)$ for $U \in \mathscr{U}$ since $\kappa(U) \subseteq \kappa(P)$ if $P \in \bar{U}$. The graph $\Gamma^{\prime}$ associated with $X_{k^{\prime}}$ is a tree, acted upon by $\operatorname{Gal}\left(k^{\prime} / k\right)$ and with the property that $\kappa\left(v^{\prime}\right)=k^{\prime}$ for every vertex $v^{\prime}$ of $\Gamma^{\prime}$. Since $\Gamma^{\prime}$ is bipartite, no two adjacent vertices can be interchanged by an element of $\operatorname{Gal}\left(k^{\prime} / k\right)$. By [Ser03, Theorem I.6.1.15], it follows that there is a vertex that is fixed by $\operatorname{Gal}\left(k^{\prime} / k\right)$. The image of this vertex under $\pi: X_{k^{\prime}} \rightarrow X$ is a vertex $v_{0} \in \mathscr{P} \cup \mathscr{U}$ of $\Gamma$ with $\kappa\left(v_{0}\right)=k$. To prove part (b), we will show that $\Gamma$ is monotonic with root $v_{0}$. For this, it suffices to show that if $v_{0}, v_{1}, \ldots, v_{n}$ are consecutive vertices of a path in $\Gamma$ (with $v_{0}$ as above), then $\kappa\left(v_{n-1}\right) \subseteq \kappa\left(v_{n}\right)$. If this does not hold, then $\kappa\left(v_{n}\right)$ is strictly contained in $\kappa\left(v_{n-1}\right)$, with $v_{n-1} \in \mathscr{P}$ and $v_{n} \in \mathscr{U}$, since $\kappa(U) \subseteq \kappa(P)$ for $P \in \mathscr{P}$ in the closure of $U \in \mathscr{U}$. Pick a path $v_{0}^{\prime}, v_{1}^{\prime}, \ldots, v_{n}^{\prime}$ in $\Gamma^{\prime}$ with $v_{i}^{\prime}$ lying over $v_{i}$; this exists because if $P \in \mathscr{P}$ lies in the closure of $U \in \mathscr{U}$, then every point of $\mathscr{P}_{k^{\prime}}$ over $P$ lies in the closure of some element of $\mathscr{U}_{k^{\prime}}$ over $U$ (that is, an irreducible component of $\left.\pi^{-1}(U)\right)$ and vice versa. Let $\sigma$ be a nontrivial element of $\operatorname{Gal}\left(k^{\prime} / \kappa\left(v_{n}\right)\right)$ that does not lie in the proper subgroup $\operatorname{Gal}\left(k^{\prime} / \kappa\left(v_{n-1}\right)\right)$. Then $\sigma$ fixes $v_{0}$ and $v_{n}$ but does not fix $v_{n-1}$; so it carries the above path to a different path in $\Gamma^{\prime}$ with the same endpoints. The concatenation of one of these paths with the inverse of the other contains a loop in $\Gamma^{\prime}$, and this gives a contradiction.

The following example shows that if $k^{\prime} / k$ is a finite field extension, then the graph $\Gamma^{\prime}$ associated with the base change $X_{k^{\prime}}$ need not be a tree even if $\Gamma$ is, when $\Gamma$ is not monotonic. The example also shows that the hypothesis in Proposition 7.6(b) is necessary. That is, it is possible in nonzero characteristic for a tree not to be monotonic, even if the graph associated with every base change of the closed fiber is a tree.

ExAmPLE 7.7. Let $k$ be a field, and let $R=k[[t]]$, with fraction field $K=k((t))$. Let $a$ be a nonsquare in $k$, and let $\mathscr{X}=\operatorname{Proj}\left(R[x, y, z] /\left((y-x)\left(x y-a z^{2}\right)+t z^{3}\right)\right)$, with function field $F$. Then $\mathscr{X}$ is a normal crossings model of $F$, whose closed fiber $X$ consists of two irreducible components $C_{1}$ and $C_{2}$, each isomorphic to $\mathbb{P}_{k}^{1}$, with $\kappa\left(C_{i}\right)=k$, and intersecting at a single point $P$ with residue field $k^{\prime}:=\kappa(P)=k(\sqrt{a})$. Thus the associated reduction graph $\Gamma$ is a tree, but it is not monotonic. If $\operatorname{char}(k) \neq 2$, then the graph $\Gamma^{\prime}$ associated with the base change $X_{k^{\prime}}$ is not a tree since $P$ splits into two points over $k^{\prime}$. But if $\operatorname{char}(k)=2$, then every base change again gives a tree since $P$ remains a single point, despite the graph not being monotonic.

\section{Examples}

As before, $K$ is a complete discretely valued field with valuation ring $R$.

In this section, we produce examples of semi-global fields $F$ over $K$ and tori $T$ for which $\amalg(F, T)$ is nontrivial or even infinite (Example 8.7). By [HHK15a, Theorem 4.2], the group $\amalg_{\mathscr{P}}(F, T)$ is trivial when $T$ is a rational $F$-torus. At the end of the section, we give an example 
of an $R$-torus for which $T \times_{R} K$ is not $K$-rational, the reduction graph has loops, and $\amalg(F, T)=0$ (Example 8.10).

A major ingredient in constructing examples where $\amalg(F, T) \neq 0$ will be Proposition 6.3 and its consequences, Theorems 6.4 and 6.5. In order to apply those results, we will want to find examples of fields $k$ and flasque $k$-tori $S$ for which $H^{1}(k, S)$ is nontrivial, or such that $H^{1}\left(k^{\prime}, S\right)$ is strictly larger than $H^{1}(k, S)$ for some finite extension $k^{\prime} / k$.

\subsection{Nontrivial $\boldsymbol{H}^{\mathbf{1}}(\boldsymbol{k}, \boldsymbol{S})$}

Let $k$ be a field, and let $L / k$ be a finite Galois extension with Galois group $G$. Let $T=R_{L / k}^{1} \mathbb{G}_{m}$ be the $k$-torus defined by the equation $\operatorname{Norm}_{L / k}(\xi)=1$. In the proof of [CTS77, Proposition 15, p. 206], an explicit flasque resolution $1 \rightarrow S \rightarrow Q \rightarrow T \rightarrow 1$ is constructed. It induces an isomorphism between $H^{1}(k, S)=T(k) / \mathrm{R}$ and $\widehat{H}^{-1}\left(G, L^{\times}\right)$. The latter is the quotient ${ }^{N} L^{\times} / I_{G} L^{\times}$ of the group ${ }^{N} L^{\times}$of norm 1 elements in $L^{\times}$by the subgroup $I_{G} L^{\times}$of $L^{\times}$generated by elements of the form $\sigma(x) / x$ for $\sigma \in G$ and $x \in L^{\times}$(see [NSW08, Section I.2]). If $L / k$ is a biquadratic Galois extension, one checks that $H^{1}(k, S)=\widehat{H}^{-1}\left(G, L^{\times}\right)$is annihilated by 2 .

EXAMPLE 8.1. Let $k$ be a field of characteristic not equal to 2 and $L=k(\sqrt{a}, \sqrt{b})$ for $a, b \in k$. Suppose $[L: k]=4$. Let $\sigma$ and $\tau$ be generators of the Galois group such that $\sigma$ fixes $\sqrt{b}$ and $\tau$ fixes $\sqrt{a}$. Suppose moreover that the quadratic form $\langle 1, a,-b\rangle=x^{2}+a y^{2}-b z^{2}$ is anisotropic over $k$. Let $T=R_{L / k}^{1}\left(\mathbb{G}_{m}\right)$. We claim that if $\sqrt{-1} \in k$, the class of $\sqrt{-1}$ in ${ }^{N} L^{\times} / I_{G} L^{\times}$is nontrivial. Indeed, first notice that $\mathrm{N}_{L / k}(\sqrt{-1})=1$, so $\sqrt{-1} \in{ }^{N} L^{\times}$. Now suppose for a contradiction that $\sqrt{-1} \in I_{G} L^{\times}$. Since $\tau \sigma(z) / z=(\sigma(z) / z)(\tau(\sigma(z)) / \sigma(z))$ for all $z \in L^{\times}$,

$$
I_{G} L^{\times}=\left\{\frac{\sigma(x)}{x} \frac{\tau(y)}{y} \mid x, y \in L^{\times}\right\} .
$$

So by assumption, $\sqrt{-1}=(\sigma(x) / x)(\tau(y) / y)$ for some $x, y \in L^{\times}$. The element $\theta:=\sigma(x) / x$ satisfies $\mathrm{N}_{L / k(\sqrt{b})}(\theta)=1$ and $\mathrm{N}_{L / k(\sqrt{a})}(\theta)=\mathrm{N}_{L / k(\sqrt{a})}(\sqrt{-1} y / \tau(y))=-1$. After writing $\theta$ as $\theta=z_{1}+z_{2} \sqrt{a}+z_{3} \sqrt{b}+z_{4} \sqrt{a b}$ with coefficients $z_{i} \in k$, these equalities on the norms give a system of equations which implies $z_{1}^{2}-a b z_{4}^{2}=0$, and hence $z_{1}=z_{4}=0$ (since $a b$ is not a square in $k$ ). Again using the system of equations, this in turn implies $b z_{3}^{2}-a z_{2}^{2}=1$. But the latter is impossible since $\langle 1, a,-b\rangle$ is anisotropic over $k$.

ExAmple 8.2. Let $L=\mathbb{Q}(\sqrt{-1}, \sqrt{2})$. Let $T=R_{L / \mathbb{Q}}^{1}\left(\mathbb{G}_{m}\right)$, and let $1 \rightarrow S \rightarrow Q \rightarrow T \rightarrow 1$ be a flasque resolution. Let $p_{i}(i \in \mathbb{N})$ be the infinite list of primes congruent to 1 modulo 8 . For any $n$, let $k_{n}:=\mathbb{Q}\left(\sqrt{p_{1}}, \ldots, \sqrt{p_{n}}\right)$. We have obvious embeddings $k_{n} \subset k_{n+1}$. Each $p_{i}$ is a square in the 2 -adic field $\mathbb{Q}_{2}$; hence there are compatible embeddings $k_{n} \subset \mathbb{Q}_{2}$. Let $k_{\infty}=\cup_{n=1}^{\infty} k_{n}$. Using class field theory, one shows that $H^{1}(\mathbb{Q}, S)=0$, that each of the groups $H^{1}\left(k_{n}, S\right)=T\left(k_{n}\right) / \mathrm{R}$ is finite of order $2^{2^{n}-1}$, see [CTS77, Corollary 2, p. 207], and that the natural maps $H^{1}\left(k_{n}, S\right) \rightarrow$ $H^{1}\left(k_{n+1}, S\right)$ are injective [Vos77, Appendix, $\S 2$, Teorema 18, p. 206]. Hence $H^{1}\left(k_{\infty}, S\right)$ is infinite.

We also give an example of an infinite first cohomology group of a flasque torus that does not rely on algebraic number theory.

Proposition 8.3. Let $1 \rightarrow S \rightarrow Q \rightarrow T \rightarrow 1$ be a flasque resolution of a $k$-torus $T$. Let $k(T)$ be the function field of $T$. If $H^{1}(k, S) \neq 0$, then the natural map $H^{1}(k, S) \rightarrow H^{1}(k(T), S)$ is injective but not surjective.

Proof. For any integral $k$-variety with a smooth $k$-point, with function field $k(X)$, and any $k$-torus $R$, the natural map $H^{1}(k, R) \rightarrow H^{1}(k(X), R)$ is injective, by an easy specialization 


\section{LOCAL-GLOBAL PRINCIPLES FOR TORI}

argument. The flasque resolution of $T$ defines a torsor over $T$ under $S$, hence defines a class $\xi \in H^{1}(T, S)$. We have the map $\delta: T(k) \rightarrow H^{1}(k, S)$ attached to the above exact sequence. For any point $P \in T(k)$, we have the equality $\delta(P)=\xi(P) \in H^{1}(k, S)$. One may also compute $\delta(P)$ by restricting the class $\xi$ to the local ring at $P$, then using the map $H^{1}\left(\mathcal{O}_{T, P}, S\right) \rightarrow H^{1}(k, S)$. Let $P \in T(k)$. We have maps

$$
H^{1}(k, S) \rightarrow H^{1}(T, S) \rightarrow H^{1}\left(\mathcal{O}_{T, P}, S\right) \rightarrow H^{1}(k(T), S) .
$$

Because $S$ is flasque, the map $H^{1}\left(\mathcal{O}_{T, P}, S\right) \rightarrow H^{1}(k(T), S)$ is an isomorphism. If the map $H^{1}(k, S) \rightarrow H^{1}(k(T), S)$ is onto, then all maps in $H^{1}(k, S) \rightarrow H^{1}\left(\mathcal{O}_{T, P}, S\right) \rightarrow H^{1}(k(T), S)$ are isomorphisms. Thus there exists a fixed $\xi_{0} \in H^{1}(k, S)$ whose image is $\xi \in H^{1}(k(T), S)$, and we get $\xi(P)=\xi_{0} \in H^{1}(k, S)$, hence $\delta(P)=\xi_{0}$. If $H^{1}(k, S) \neq 0$, since the map $T(k) \rightarrow H^{1}(k, S)$ is onto, there exist $k$-points $P$ and $Q$ such that $\delta(P) \neq \delta(Q) \in H^{1}(k, S)$. Thus $H^{1}(k, S) \neq 0$ implies that the natural map $H^{1}(k, S) \rightarrow H^{1}(k(T), S)$ is not onto.

EXAMPLE 8.4. Let $k$ be a field, and let $S$ be a flasque torus over $k$ with $H^{1}(k, S) \neq 0$. One may then produce an exact sequence of tori $1 \rightarrow S \rightarrow Q \rightarrow T \rightarrow 1$ with $Q$ quasitrivial, by letting $\widehat{T}$ be the kernel of an equivariant surjection from a permutation module to the character group $\widehat{S}$. Using Proposition 8.3, passing from $k$ to $k(T)$ and iterating the process (with the same exact sequence), we produce a field $E$ of infinite transcendence degree over $k$ such that $H^{1}(E, S)$ is infinite.

EXAMPLE 8.5. Let $k=\mathbb{R}((x))((y))$ and $L=k(\sqrt{x}, \sqrt{y})$. Let $T=R_{L / k}^{1}\left(\mathbb{G}_{m}\right)$, and let $1 \rightarrow$ $S \rightarrow Q \rightarrow T \rightarrow 1$ be a flasque resolution of $T$. Let $k^{\prime}=\mathbb{C}((x))((y))$. Then one can show that $H^{1}(k, S)=0$ and $H^{1}\left(k^{\prime}, S\right) \neq 0$. In particular, the map $H^{1}(k, S) \rightarrow H^{1}\left(k^{\prime}, S\right)$ is not onto.

Proposition 8.6. Let $k$ be a local field of characteristic equal to 2. Then $k=\mathbb{F}((s))$ for some finite field $\mathbb{F}$ with char $(\mathbb{F})=2$. Let $k^{\prime}=k(\sqrt{s})$. Let $L / k$ be the biquadratic Galois extension of $k$ with Galois group $G=\mathbb{Z} / 2 \times \mathbb{Z} / 2$, and let $T=R_{L / k}^{1} \mathbb{G}_{m}$. Let $1 \rightarrow S \rightarrow Q \rightarrow T \rightarrow 1$ be a flasque resolution of $T$. Then the map $H^{1}(k, S) \rightarrow H^{1}\left(k^{\prime}, S\right)$ is not onto.

Proof. Recall $H^{1}(k, S) \simeq{ }^{N} L^{*} / I_{G} L^{*}$. Let $\alpha \in L^{*}$ with $N_{L / k}(\alpha)=1$. Since $k$ is a local field of characteristic 2, we have an inclusion $L^{*} \subset L(\sqrt{s})^{* 2}$. Hence $\alpha=\beta^{2}$ for some $\beta \in L(\sqrt{s})=L k^{\prime}$. Since $N_{L / k}(\alpha)=1$, we have $N_{L k^{\prime} / k^{\prime}}(\beta)^{2}=1$. Since $\operatorname{char}(k)=2$, we have $N_{L k^{\prime} / k^{\prime}}(\beta)=1$. Thus $\beta$ gives rise to an element in $H^{1}\left(k^{\prime}, S\right)$. Since $H^{1}\left(k^{\prime}, S\right)$ is 2-torsion, $\alpha=\beta^{2}$ is trivial in $H^{1}\left(k^{\prime}, S\right)$. Thus the natural map $H^{1}(k, S) \rightarrow H^{1}\left(k^{\prime}, S\right)$ is the trivial map. But $H^{1}\left(k^{\prime}, S\right)=\widehat{H}^{-1}\left(G,\left(L k^{\prime}\right)^{*}\right)$; by [NSW08, Theorem 7.2.1], the latter group is dual to $\widehat{H}^{3}(G, \mathbb{Z})=H^{3}(G, \mathbb{Z}) \simeq \mathbb{Z} / 2$. Thus the map $H^{1}(k, S) \rightarrow H^{1}\left(k^{\prime}, S\right)$ is not onto.

\subsection{Nontrivial $\amalg(F, T)$}

Let $T$ be a smooth $R$-torus, and let $F$ be a semi-global field over the fraction field of $R$. In Theorem 7.3, we proved that $\amalg(F, T)$ is trivial if the reduction graph of a normal crossings model of $F$ is a monotonic tree. In Examples 8.7 and 8.8, we show that if the reduction graph is not a tree, then $\amalg(F, T)$ can behave quite differently. Afterwards, in Example 8.9, we show that a nonmonotonic tree can also lead to a nontrivial $\amalg(F, T)$.

ExAmple 8.7. Let $k$ be a field, and let $R=k[[t]]$ with fraction field $K=k((t))$. Let $\mathscr{X}=$ $\operatorname{Proj}\left(R[x, y, z] /\left(x y z-t(x+y+z)^{3}\right)\right)$, and let $F$ be the function field of $\mathscr{X}$. Then $\mathscr{X}$ is a regular normal crossings model of $F$, whose (reduced) closed fiber $X$ consists of three irreducible components $C_{1}, C_{2}, C_{3}$, each isomorphic to $\mathbb{P}_{k}^{1}$, with $\kappa\left(C_{i}\right)=k$, any two of which meet transversely at a single $k$-point. The reduction graph $\Gamma$ is a triangle, hence not a tree. It satisfies $H_{1}\left(\Gamma_{\text {top }}, \mathbb{Z}\right)=\mathbb{Z}$. 


\section{JLCT, DH, JH, DK, RP AND VS}

Let $T$ be a $k$-torus, and let $1 \rightarrow S \rightarrow Q \rightarrow T \rightarrow 1$ be a flasque resolution. Then by Theorem 6.4(c), we have $\amalg(F, T) \simeq H^{1}(k, S)$. If $k$ and $T$ are as in Example 8.1 or as in Example 8.2, then $\amalg(F, T) \neq 0$. If $k$ and $T$ are as in Example 8.2, then $\amalg(F, T)$ is infinite. Similarly, Example 8.4 can be used to produce an example of infinite $\amalg(F, T)$ (with residue field $k=E)$.

We now give an example of a semi-global field $F$ and an $R$-torus with $\amalg(F, T)$ trivial and $\amalg(L, T)$ nontrivial for some finite field extension $L / F$ (which in fact comes from an extension of $k)$.

ExAmple 8.8. Let $k$ be a field, let $R=k[[t]]$, and let $\mathscr{X}$ and $F$ be as in Example 8.7. Let $T$ be a $k$-torus, and let $1 \rightarrow S \rightarrow Q \rightarrow T \rightarrow 1$ be a flasque resolution. Then by Theorem 6.4(c), we have $\amalg(F, T) \simeq H^{1}(k, S)$. Now let $k, T$, and $k^{\prime}$ be as in Example 8.5; or let $k=\mathbb{Q}$, let $T$ be as in Example 8.2, and let $k^{\prime}=\mathbb{Q}(\sqrt{17})$. Then $\amalg(F, T) \simeq H^{1}(k, S)=0$ and $\amalg\left(F \otimes_{k} k^{\prime}, T\right) \simeq$ $H^{1}\left(k^{\prime}, S\right) \neq 0$.

We next give two related examples to show that $\amalg(F, T)$ can be nontrivial even when the reduction graph is a tree, if it is not a monotonic tree. As we saw in Proposition 7.6, a reduction graph that is a tree can fail to be monotonic if the reduction graph of some base change is not a tree, or if the residue field of $R$ has finite characteristic. Our two examples illustrate each of those two situations.

EXAmple 8.9. Let $T$ be a torus over a field $k$, let $R=k[[t]]$, and let $\mathscr{X}$ and $F$ be as in Example 7.7. We consider two cases:

(a) Let $k, T$, and $k^{\prime}$ be as in Example 8.5, so that $k$ is of characteristic zero. As noted in Example 7.7 , the reduction graph $\Gamma$ of $\mathscr{X}$ is a tree but not a monotonic tree since the reduction graph of the base change to $k^{\prime}$ is not a tree. Since $H_{1}\left(\Gamma_{\text {top }}, \mathbb{Z}\right)=0$, Theorem 6.5 yields $\amalg(F, T) \simeq H^{1}\left(k^{\prime}, S\right) / H^{1}(k, S) \neq 0$.

(b) Let $k, T$, and $k^{\prime}$ be as in Proposition 8.6, so that $k$ has characteristic 2 . The reduction graph $\Gamma$ of $\mathscr{X}$ is a tree but not a monotonic tree, even though in this case the reduction graph of every base change is still a tree. Again we have $\amalg(F, T) \simeq H^{1}\left(k^{\prime}, S\right) / H^{1}(k, S) \neq 0$.

In [HHK15a, Theorem 5.10, Corollary 6.5], it was shown that if $G$ is a connected linear algebraic group over a semi-global field $F$, and if $G$ is rational as an $F$-variety, then $\amalg_{\mathscr{P}}(F, G)=$ $\amalg_{X}(F, G)$ is trivial, whether or not the reduction graph is a tree. This prompts the following two questions in our situation:

First, given a $k$-torus $T$ and a semi-global field $F$ over $k((t))$, must $\amalg(F, T)=\amalg_{\mathscr{P}}(F, T)$ be trivial if the reduction graph of a normal crossings model of $F \times_{k} k^{\prime}$ is a tree, where $k^{\prime}$ is a splitting field of $T$ (in other words, is such that $T_{k^{\prime}}$ is a split torus and hence rational)? Example 8.9(b) shows that the answer to that question is no. There, the field $L$ in Proposition 8.6 is a splitting field of $T$, but the reduction graph of the base change to $L$ (and indeed to any finite extension of $k$ ) is a tree.

Second, if a torus $T$ is defined over $R$ but is not rational, and if the reduction graph associated with a normal crossings model of $F$ is not a tree, can $\amalg(F, T)$ still be trivial? The example in the next section shows that the answer is yes.

\subsection{Failure of the exact sequence for nonrational components}

Let $\mathscr{X}$ be a normal crossings model of a semi-global field $F$ over a complete discretely valued field $K$ with residue field $k$ and valuation ring $R$. Let $\mathscr{P}$ be the set of intersection points of 


\section{LOCAL-GLOBAL PRINCIPLES FOR TORI}

the components of the closed fiber of $\mathscr{X}$, and let $\Gamma$ be the associated reduction graph. Let $T$ be an $R$-torus with flasque resolution $1 \rightarrow S \rightarrow Q \rightarrow T \rightarrow 1$. If the closed fiber consists of a union of copies of $\mathbb{P}_{k}^{1}$ meeting at $k$-points, then by Theorem 6.4(c), we have an isomorphism $\amalg(F, T) \simeq H^{1}(k, S)^{m}$, where $m$ is the rank of $H_{1}\left(\Gamma_{\text {top }}, \mathbb{Z}\right)$.

We now give an example to show that we need not have such an isomorphism if some irreducible component of $X$ is not isomorphic to $\mathbb{P}_{k}^{1}$. In fact, this example will also show that $\amalg(F, T)$ can be trivial for a nonrational torus even if the reduction graph is not a tree. Therefore, $\amalg(F, T)$ can vanish even if this would not be predicted by that isomorphism. In this example, it will be convenient to use R-equivalence; see Section 2.

EXAmple 8.10. Let $k=k_{1}, T, S$ be as in Example 8.2 (with $n=1$ ). Then $\left|H^{1}(k, S)\right|=$ $|T(k) / \mathrm{R}|=2$. In particular, $T$ is not rational. Let $g \in T(k)$ be a representative of the nontrivial class. Let $C / k$ be a smooth curve with a rational map $f: C \rightarrow T$ defined at points $Q_{1}, Q_{2} \in C(k)$ such that $f\left(Q_{1}\right)=1 \in T(k)$ and $f\left(Q_{2}\right)=g$. Let $C^{*}$ be the nodal curve obtained from $C$ by identifying $Q_{1}$ and $Q_{2}$. By [HS99, Theorem 3], there is a regular integral proper curve $\mathscr{X} / k[[t]]=R$ with closed fiber $C^{*}$ since the node can be deformed locally to the spectrum of a regular complete local ring of dimension 2. Let $P$ be the image of $Q_{1}$ and $Q_{2}$ in $C^{*}$. Blowing up $\mathscr{X}$ at $P$ gives a normal crossings model whose closed fiber is a union of $\mathbb{P}_{k}^{1}$ and $C$, meeting at the two $k$-points $Q_{1}$ and $Q_{2}$. Let $U$ be the complement of $\left\{Q_{1}, Q_{2}\right\}$ in $\mathbb{P}_{k}^{1}$, let $V$ be the complement of $\left\{Q_{1}, Q_{2}\right\}$ in $C$, and let $\Gamma$ be the associated reduction graph. Then by Lemma 6.2, we have $\amalg(F, T)=H^{1}\left(\Gamma, \mathcal{H}_{k}(S)\right)$. Recall that the coefficient system $\mathcal{H}_{k}(S)$ is given by decorating $Q_{i}$ with $H^{1}(k, S) \simeq T(k) / \mathrm{R}$, decorating $U$ with $H^{1}(k(U), S)=H^{1}(k, S) \simeq T(k) / \mathrm{R}$, and decorating $V$ with $H^{1}(k(V), S) \simeq T(k(V)) /$ R. Here the isomorphism $H^{1}(k(U), S)=H^{1}(k, S)$ holds because $U$ is an affine open subset of $\mathbb{P}_{k}^{1}$, as in the proof of Theorem 6.5.

Now view $f$ above as an element in $T(k(V))=T(k(C))$. By definition, the specialization maps on $V$ at $Q_{1}$ and $Q_{2}$ send $f$ to 1 and to the class of $g$, respectively. It is then easy to see that in the corresponding cochain complex, $\mathcal{C}^{0} \rightarrow \mathcal{C}^{1}$ is onto, and hence $\amalg(F, T)=0$. But $\Gamma$ has a loop and $H^{1}(k, S) \neq 0$, so $\operatorname{Hom}\left(H_{1}\left(\Gamma_{\text {top }}, \mathbb{Z}\right), H^{1}(k, S)\right) \neq 0$.

Note that Example 8.10 also produces an example for the triviality of $\amalg(F, T)$ even though the closed fiber of an associated normal crossings model is not a tree and $T$ is not rational.

\section{REFERENCES}

CTGP04 J.-L. Colliot-Thélène, P. Gille, and R. Parimala, Arithmetic of linear algebraic groups over 2dimensional geometric fields, Duke Math. J. 121 (2004), no. 2, 285-341; doi:10.1215/S00127094-04-12124-4.

CTHH $^{+} 19$ J.-L. Colliot-Thélène, D. Harbater, J. Hartmann, D. Krashen, R. Parimala, and V. Suresh, Local-global principles for zero-cycles on homogeneous spaces over arithmetic function fields, Trans. Amer. Math. Soc. 372 (2019), no. 8, 5263-5286; doi:10.1090/tran/7911.

CTPS12 J.-L. Colliot-Thélène, R. Parimala, and V. Suresh, Patching and local-global principles for homogeneous spaces over function fields of p-adic curves, Comment. Math. Helv. 87 (2012), no. 4, 1011-1033; doi:10.4171/CMH/276.

CTPS16_L Lois de réciprocité supérieures et points rationnels, Trans. Amer. Math. Soc. $\mathbf{3 6 8}$ (2016), no. 6, 4219-4255; doi:10.1090/tran/6519.

CTS77 J.-L. Colliot-Thélène and J. J. Sansuc, La R-équivalence sur les tores, Ann. Sci. École Norm. Sup. (4) 10 (1977), no. 2, 175-229; doi:10.24033/asens.1325. 
CTS87 _ Principal homogeneous spaces under flasque tori: applications, J. Algebra 106 (1987), no. 1, 148-205; doi:10.1016/0021-8693(87)90026-3.

Dem65 M. Demazure, Automorphismes des groupes réductifs, Exposé XXIV in Schémas en Groupes (Séminaire de Géométrie Algébrique du Bois Marie 1962-1964 (SGA3), Tome 3), Revised and annotated edition of the 1970 French original, Doc. Math., vol. 8 (eds P. Gille and P. Polo; Soc. Math. France, Paris, 2011).

DM69 P. Deligne and D. Mumford, The irreducibility of the space of curves of given genus, Publ. Math. Inst. Hautes Études Sci. 36 (1969), 75-109; doi:10.1007/BF02684599.

Gro61 A. Grothendieck, Éléments de géométrie algébrique. III. Étude cohomologique des faisceaux cohérents. I, Publ. Math. Inst. Hautes Études Sci. 11 (1961), 5-159; doi:10.1007/ $\mathrm{BF} 02684273$.

HHK09 D. Harbater, J. Hartmann, and D. Krashen, Applications of patching to quadratic forms and central simple algebras, Invent. Math. 178 (2009), no. 2, 231-263; doi:10.1007/s00222-0090195-5.

HHK11_ Patching subfields of division algebras, Trans. Amer. Math. Soc. 363 (2011), no. 6, 3335-3349; doi:10.1090/S0002-9947-2010-05229-8.

HHK15a , Local-global principles for torsors over arithmetic curves, Amer. J. Math. 137 (2015), no. 6, 1559-1612; doi:10.1353/ajm.2015.0039.

HHK15b_ Refinements to patching and applications to field invariants, Int. Math. Res. Not. 2015 (2015), no. 20, 10399-10450; doi:10.1093/imrn/rnu278.

HS99 D. Harbater and K. F. Stevenson, Patching and thickening problems, J. Algebra 212 (1999), no. 1, 272-304; doi:10.1006/jabr.1998.7574.

HS16 D. Harari and T. Szamuely, Local-global questions for tori over p-adic function fields, J. Algebraic Geom. 25 (2016), no. 3, 571-605; doi:10.1090/jag/661.

Hu17 Y. Hu, A cohomological Hasse principle over two-dimensional local rings, Int. Math. Res. Not. 2017 (2017), no. 14, 4369-4397; doi:10.1093/imrn/rnw149.

Lan56 S. Lang, Algebraic groups over finite fields, Amer. J. Math. 78 (1956), 555-563; doi:10.2307/ 2372673.

Lic68 S. Lichtenbaum, Curves over discrete valuation rings, Amer. J. Math. 90 (1968), 380-405; doi:10.2307/2373535.

Lip75 J. Lipman, Introduction to resolution of singularities, Algebraic Geometry (Humboldt State Univ., Arcata, Calif., 1974), Proc. Sympos. Pure Math., vol. 29 (Amer. Math. Soc., Providence, RI, 1975), 187-230.

Lip78 _ Desingularization of two-dimensional schemes, Ann. Math. 107 (1978), no. 1, 151207; doi:10.2307/1971141.

Mil80 J. S. Milne, Étale cohomology, Princeton Math. Ser., vol. 33 (Princeton Univ. Press, Princeton, NJ, 1980).

NSW08 J. Neukirch, A. Schmidt, and K. Wingberg, Cohomology of number fields, 2nd ed., Grundlehren math. Wiss., vol. 323 (Springer-Verlag, Berlin, 2008); doi:10.1007/978-3-540-37889-1.

PR94 V. Platonov and A. Rapinchuk, Algebraic groups and number theory, Pure Appl. Math., vol. 139 (Academic Press, Inc., Boston, MA, 1994).

Ser03 J.-P. Serre, Trees, Springer Monogr. Math. (Springer-Verlag, Berlin, 2003); doi: 10.1007/9783-642-61856-7.

Str83 R. Strano, Principal homogeneous spaces over Hensel rings, Proc. Amer. Math. Soc. 87 (1983), no. 2, 208-212; doi:10.2307/2043691.

Vos77 V. E. Voskresenskiŭ, Algebraicheskie tory (Algebraic tori; Russian) (Izdat. "Nauka", Moscow, 1977). 
Jean-Louis Colliot-Thélène jlct@math.u-psud.fr

Université Paris-Saclay, CNRS, Laboratoire de Mathématiques d'Orsay, 91405, Orsay, France

David Harbater harbater@math.upenn.edu

Department of Mathematics, University of Pennsylvania, Philadelphia, PA 19104-6395, USA

Julia Hartmann hartmann@math.upenn.edu

Department of Mathematics, University of Pennsylvania, Philadelphia, PA 19104-6395, USA

Daniel Krashen daniel.krashen@rutgers.edu

Department of Mathematics, Rutgers University, Piscataway, NJ 08854-8019, USA

Raman Parimala parimala.raman@emory.edu

Department of Mathematics and Computer Science, Emory University, Atlanta, GA 30322, USA

Venapally Suresh suresh.venapally@emory.edu

Department of Mathematics and Computer Science, Emory University, Atlanta, GA 30322, USA 